\title{
Spectroscopic classification of red high proper motion objects in the Southern Sky ${ }^{\star, \star \star}$
}

\author{
N. Lodieu ${ }^{1,2}$, R.-D. Scholz ${ }^{1}$, M. J. McCaughrean ${ }^{1,3}$, R. Ibata ${ }^{4}$, M. Irwin ${ }^{5}$, and H. Zinnecker ${ }^{1}$ \\ 1 Astrophysikalisches Institut Potsdam, An der Sternwarte 16, 14482 Potsdam, Germany \\ 2 Department of Physics \& Astronomy, University of Leicester, University Road, Leicester LE1 7RH, UK \\ e-mail: nl41@star.le.ac.uk \\ ${ }^{3}$ School of Physics, University of Exeter, Stocker Road, Exeter EX4 4QL, UK \\ ${ }^{4}$ Observatoire de Strasbourg, 11 rue de l'Université, 67000 Strasbourg, France \\ ${ }^{5}$ Institute of Astronomy, Madingley Road, Cambridge CB3 0HA, UK
}

Received 30 November 2004 / Accepted 6 June 2005

\begin{abstract}
We present the results of spectroscopic follow-up observations for a sample of 71 red objects with high proper motions in the range $0.08-1.14 \operatorname{arcsec}_{\mathrm{yr}^{-1}}$ as detected using APM and SSS measurements of multi-epoch photographic Schmidt plates. Red objects were selected by combining the photographic $B_{J} R I$ magnitudes with 2MASS near-infrared $J H K_{\mathrm{s}}$ magnitudes. Some 50 of the 71 spectroscopically classified objects turn out to be late-type (>M6) dwarfs and in more detail, the sample includes 35 ultracool dwarfs with spectral types between M8 and L2, some previously reported, as well as five M-type subdwarfs, including a cool esdM6 object, SSSPM J0500-5406. Distance estimates based on the spectral types and 2MASS $J$ magnitudes place almost all of the late-type ( $>$ M6) dwarfs within $50 \mathrm{pc}$, with 25 objects located inside the $25 \mathrm{pc}$ limit of the catalogue of nearby stars. Most of the early-type M dwarfs are located at larger distances of 100-200 pc, suggesting halo kinematics for some of them. All objects with $\mathrm{H} \alpha$ equivalent widths larger than $10 \AA$ have relatively small tangential velocities $\left(<50 \mathrm{~km} \mathrm{~s}^{-1}\right)$. Finally, some late-type but blue objects are candidate binaries.
\end{abstract}

Key words. surveys - stars: kinematics - stars: late-type - stars: low-mass, brown dwarfs - techniques: photometric techniques: spectroscopic

\section{Introduction}

Although red dwarf stars of spectral type "M" account for more than $70 \%$ of the stellar number density in the Solar neighbourhood and are estimated to contribute nearly half of the total stellar mass in the Galaxy (Henry et al. 1997), they are not easily detectable in magnitude-limited sky surveys. Indeed, not a single known $\mathrm{M}$ dwarf, including the nearest star, Proxima Centauri (M5, $d=1.3 \mathrm{pc}, V=11.1 \mathrm{mag}$ ), or the nearest M0 dwarf, AX Microscopium ( $d=3.9 \mathrm{pc}, V=6.7 \mathrm{mag})$, is visible to the naked eye. Wide-field, deep images taken with Schmidt telescopes preferentially reveal distant main sequence stars of earlier spectral types and more distant giants, but relatively few $M$ dwarfs per field. One way of identifying these nearby, cool, very low luminosity dwarf stars and their substellar counterparts is to compare multi-wavelength sky survey data and select on their characteristic colours. The drawback,

* Based on observations collected with the VLT/FORS1 and ISAAC, the ESO 3.6-m/EFOSC2 and the NTT/SOFI at the European Southern Observatory, Paranal and La Silla, Chile (ESO programmes 63.L-0634, 65.L-0689, 68.C-0664, and 70.C-0568).

$\star \star$ Tables 1-4 are only available in electronic form at http://www. edpsciences.org however, is the presence of Galactic halo and compact extragalactic sources with similar colours.

Proper motion surveys are an effective way to discriminate between distant giants and nearby dwarfs with similar colours.

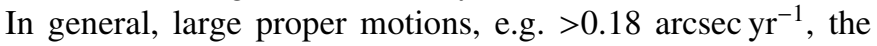
lower limit in the New Luyten Two Tenths (NLTT) catalogue of $\sim 60000$ stars (Luyten 1979-80), indicate either small distances $(d<50$ pc for members of the Galactic disk population with typical tangential velocities $v_{\mathrm{t}}$ of about $40 \mathrm{~km} \mathrm{~s}^{-1}$ ) and/or intrinsic high velocities $(d<180 \mathrm{pc}$ for Galactic halo members with typical $v_{\mathrm{t}} \sim 150 \mathrm{~km} \mathrm{~s}^{-1}$ ) (Scholz et al. 2000). As a consequence, proper motion samples are highly biased towards halo members: according to Digby et al. (2003), the ratio of disk to halo stars can be as low as $4: 1$ for a proper motion-limited sample, compared to 400:1 for a true Solar neighbourhood volume-limited sample. On the other hand, it is easier to rule out distant (halo) red giants in a sample of faint (e.g. $R>13$ ), red objects with proper motions exceeding the NLTT limit, since the proper motion would imply tangential velocities larger than $v_{\mathrm{t}}>10000 \mathrm{~km} \mathrm{~s}^{-1}$ at distances of $d>12 \mathrm{kpc}$, a factor of 20 larger than the Galactic escape speed (cf. Leonard \& Tremaine 1990; Meillon et al. 1997). 
Despite the advantages of the high proper motion approach, the 3-D picture of the Solar neighbourhood is remarkably incomplete for low luminosity dwarfs due to the limiting magnitude and incompleteness of existing high proper motion catalogues, particularly in the southern sky and in the Galactic plane region. Within the $25 \mathrm{pc}$ horizon of the catalogue of nearby stars (Gliese \& Jahreiß 1991), possibly as many as 63\% of stellar systems remain undiscovered (Henry et al. 2002), while the number of missing systems within $10 \mathrm{pc}$ is likely to be more than $30 \%$ according to Henry et al. (1997). Their prediction for the $10 \mathrm{pc}$ sample of $\sim 130$ missing systems compared to the 229 known ones is based on the assumptions (1) that the density of stellar systems within $5 \mathrm{pc}\left(0.084\right.$ systems $\left.\mathrm{pc}^{-3}\right)$ extends to $25 \mathrm{pc}$; and (2) that the distribution of systems is isotropic. By comparing their $8 \mathrm{pc}$ sample to the $10 \mathrm{pc}$ sample, Reid et al. (2003) estimated an incompleteness in the latter of about $25 \%$, with most of the missing stars are expected among the late-type $\mathrm{M}$ dwarfs. Even the $5 \mathrm{pc}$ sample cannot yet be considered complete with respect to these ultracool M dwarfs, as was recently demonstrated by the discovery of an M9 dwarf at about 4 pc (Delfosse et al. 2001) and an M6.5 dwarf at about $3 \mathrm{pc}$ (Teegarden et al. 2003).

Several groups have contributed to the completion of the nearby sample in recent years. Since the identification of the twentieth nearest star, GJ 1061 (Henry et al. 1997), about 20 new $\mathrm{M}$ dwarfs have been discovered within $10 \mathrm{pc}$. These include mid-M (M3.5 to M6.5) dwarfs (Scholz et al. 2001; Reid \& Cruz 2002; Reid et al. 2002a; Reylé et al. 2002; Henry et al. 2002; Phan-Bao et al. 2003; Teegarden et al. 2003) and late-type (M7 to M9.5) dwarfs (Gizis et al. 2000; Delfosse et al. 2001; McCaughrean et al. 2002; Reid et al. 2003; Lépine et al. 2003c). Many of the mid-M dwarfs and some of the lateM dwarfs had already been catalogued as proper motion stars by Luyten (the Luyten Half Second (LHS) catalogue for stars with proper motions exceeding $0.5 \operatorname{arcsec} \mathrm{yr}^{-1}$; Luyten 1979), but their distances had not been established. Initial distance estimates are generally arrived at via spectroscopic or photometric measurements, although these can be misleading if the sources are multiples. Ultimately, confirmation via trigonometric parallax measurements is required.

Thanks to new near-infrared sky surveys, including the Two-Micron All Sky Survey (2MASS; Skrutskie et al. 1997) and the DEep Near-Infrared Survey (DENIS; Epchtein et al. 1997), and deep optical CCD surveys, including the Sloan Digital Sky Survey (SDSS; York et al. 2000), large numbers of objects even cooler than the latest-type $\mathrm{M}$ dwarfs have been recently discovered. These have led to the definition of two new spectral types, $\mathrm{L}$ and $\mathrm{T}$, with accurate optical and nearinfrared classification schemes (Kirkpatrick et al. 1999; Martín et al. 1999; Burgasser et al. 2002; Geballe et al. 2002). Many of these sources are also nearby with high proper motions including, for example, the $\mathrm{T}$ dwarf binary $\varepsilon$ Indi $\mathrm{Ba}, \mathrm{Bb}$ at $3.6 \mathrm{pc}$, which has a proper motion of $4.8 \operatorname{arcsec}_{\mathrm{yr}^{-1}}$ (Scholz et al. 2003; McCaughrean et al. 2004).

In this paper, we present the results of various photometric and spectroscopic follow-up observations of very red, high proper motion stars in the southern sky, selected as ultracool dwarf candidates during different stages of our ongoing high proper motion survey. The paper is organised as follows: Sect. 2 describes the various sample selection methods applied to reveal low-mass neighbours to the Sun. Section 3 outlines the optical and near-infrared photometry and spectroscopy observations made with several ESO telescopes, including the Very Large Telescope (VLT), New Technology Telescope (NTT), and 3.6- $\mathrm{m}$. Section 4 explains the procedures employed in classifying our targets based on their optical and near-infrared properties. A subsample of low-metallicity subdwarfs found in the course of the proper motion survey is presented in Sect. 5 . We discuss distance estimates in Sect. 6, as well as the kinematics and measures of activity in Sect. 7, for all objects detected in our search. Individual objects with specific properties are discussed in more detail in Sect. 8, including L dwarfs previously published by Lodieu et al. (2002) and Scholz \& Meusinger (2002), and M dwarfs reported by McCaughrean et al. (2002).

\section{Selection of red proper motion stars}

Detecting proper motion stars requires observational data spanning an appropriately long period and the original Palomar Sky Survey (POSS-1) led to the discovery of many proper motion sources when it was compared with newer plate material. However, the relatively poor sensitivity of the POSS-1 plates meant that they were unable to pick up the very red and relatively faint $\mathrm{L}$ and $\mathrm{T}$ dwarfs: to date, none of the NLTT stars has been found to be an L dwarf or later. However, the more modern POSS-2, UK Schmidt, and ESO Schmidt surveys provided substantial improvements in sensitivity at the red end over several epochs, making it possible to discover nearby, high proper motion $\mathrm{L}$ and $\mathrm{T}$ dwarfs via photographic material alone. This was successfully demonstrated by the discovery of the first field L dwarf, Kelu 1 (Ruiz et al. 1997), other bright L dwarfs including SSSPM J0829-1309 (Scholz \& Meusinger 2002) and LSR 0602+3910 (Salim et al. 2003), and the very nearby $\mathrm{T}$ dwarfs $\varepsilon$ Indi Ba,Bb (Scholz et al. 2003). Finally, comparison of data from digital optical and infrared sky surveys such as SDSS, 2MASS, and DENIS, with earlier plate material and/or second epoch digital data has also led to the discovery of large numbers of $\mathrm{L}$ and $\mathrm{T}$ dwarfs (Kirkpatrick et al. 1999, 2000; Reid et al. 2000; Scholz \& Meusinger 2002; Tinney et al. 2003; Burgasser et al. 2003b,c, 2004). All the nearest late- $\mathrm{L}$ and $\mathrm{T}$ dwarfs have also been confirmed as large proper motion objects (Dahn et al. 2002; Vrba et al. 2004).

For the present work, we chose to use archival multi-epoch optical photographic plate material to search for new high proper motion sources, in common with some other groups. Several searches have been made in the poorly investigated southern sky (e.g., Wroblewski \& Torres 1994; Wroblewski \& Costa 1999; Scholz et al. 2000; Ruiz et al. 2001; Reylé et al. 2002; Pokorny et al. 2003; Hambly et al. 2004), while new, thorough surveys have also been made in the northern sky (Lépine et al. 2002) and in the Galactic plane (Lépine et al. 2003d).

Here, we started by selecting red candidates from the high proper motion survey initiated by Scholz et al. (2000) to uncover missing nearby low-mass stars and brown dwarfs. This survey was originally based on APM (Automatic Plate 


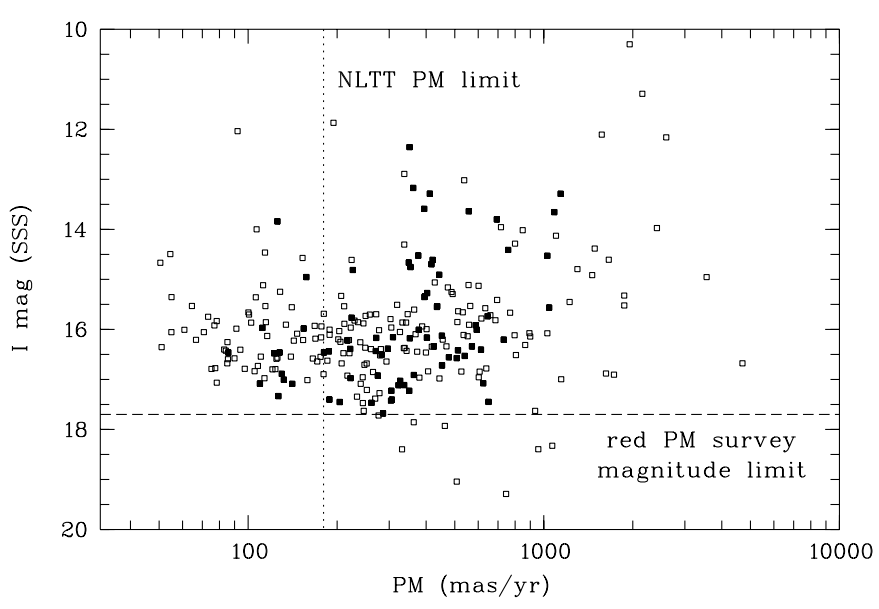

Fig. 1. $I$-band magnitudes and proper motions for our full sample of about 270 red proper motion objects: the filled symbols represent the objects described in the current paper (Table 1). The handful of objects shown below the red proper motion survey magnitude limit (dashed line) were originally discovered and classified as $\mathrm{L}$ and $\mathrm{T}$ dwarfs in deep digital surveys (SDSS, 2MASS, DENIS) based on their colours, but were then recovered a posteriori in the SSS photographic data.

Measuring) machine scans of UKST plates in two passbands $\left(B_{J}\right.$ and $R$ ) covering a few thousand square degrees (see, e.g., Reylé et al. 2002): high proper motion sources discovered in this work are given the prefix APMPM. More recently, the survey was extended to use the multi-epoch astrometry and multicolour $\left(B_{J}, R, I\right)$ photographic photometry data in the first release of the SuperCOSMOS Sky Surveys (SSS; Hambly et al. 2001a,b,c): sources found this way are prefixed SSSPM and the present sample derived from our recently completed southern sky SSS survey is shown in Fig. 1. It should be noted in passing that the same SSS data are being used completely independently for the same purpose by other groups (Pokorny et al. 2003; Hambly et al. 2004).

Our strategies for selecting nearby, ultracool dwarfs from the photographic plate catalogues are constantly evolving and being refined, employing different combinations of proper motions, colours, magnitudes, and so on, as new photometric data become available and as our experience with spectroscopically confirmed sources is folded back into the procedure. The main search strategies employed to date can be summarised as follows (in chronological order):

1. APM data were available for only one pair of plates $\left(B_{J}\right.$ and $R$ ) per field, with a typical epoch difference between 5 and 15 years. We selected objects shifted by more than 5 arcsec (corresponding to a lower proper motion limit

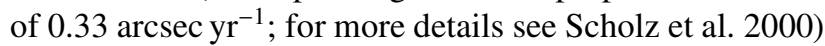
and with $B_{J}-R>2.5$. Since the photographic $B_{J}-R$ colour is only weakly correlated with spectral type (see Fig. 2, cf. Fig. 3 in Kirkpatrick et al. 1997), it was hard to predict the optical and near-infrared fluxes for each source and thus we obtained optical $(R, I)$ and near-infrared $\left(J, H, K_{\mathrm{s}}\right)$ photometry from VLT pre-imaging in order to ensure accurate exposure times for the subsequent spectroscopy (see Sect. 3.1).
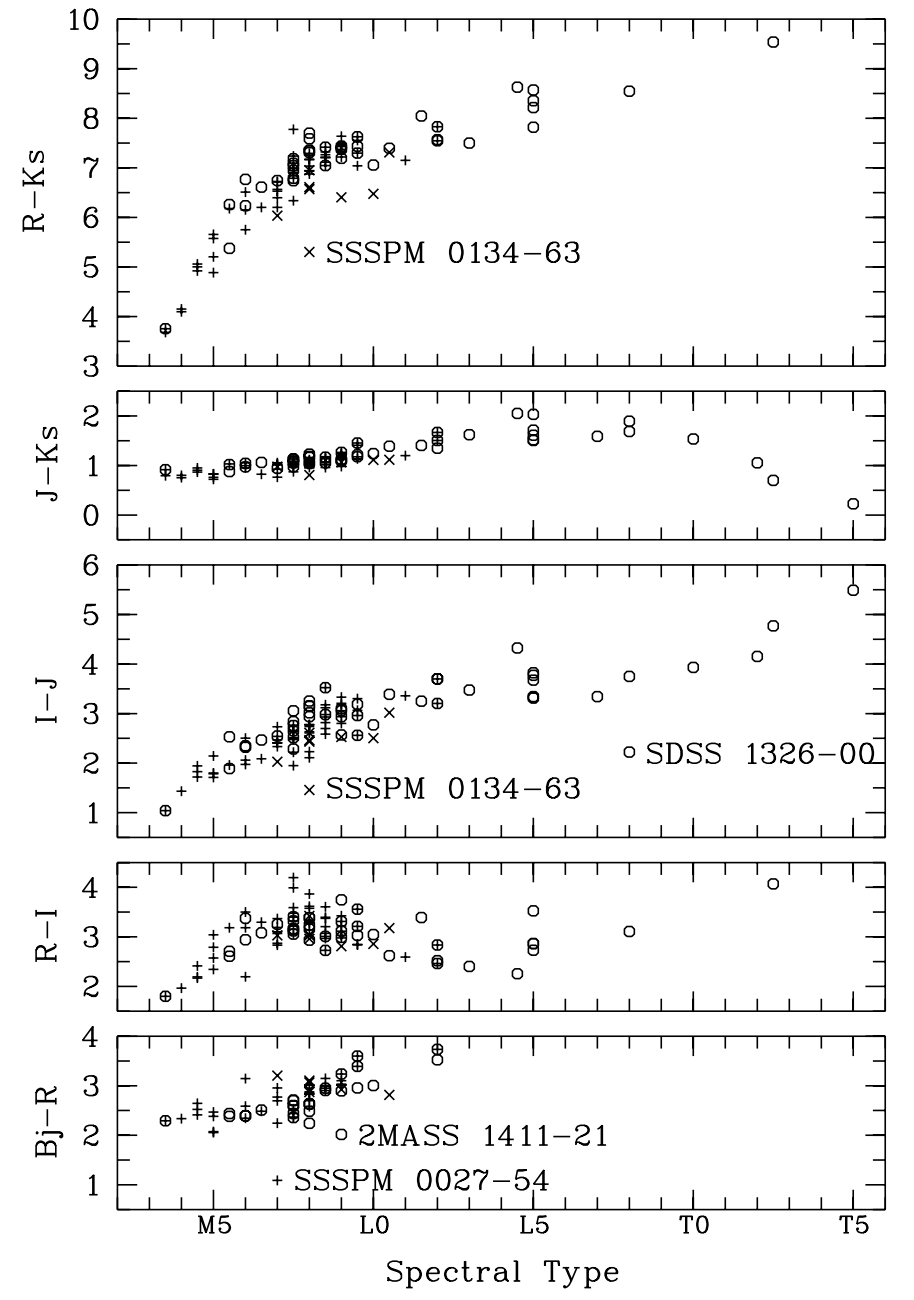

Fig. 2. Colours of late M, L and T dwarfs as derived from SSS optical photometry $\left(B_{J}, R, I\right)$ and 2MASS near-infrared photometry $\left(J, K_{\mathrm{s}}\right)$. Only objects detected in SSS data with spectral types obtained in this study (+ optical, $\times$ near-infrared) and from the literature (open circles) are shown. Outliers with atypically blue colours for their spectral types are labelled by name (see Sect. 8.4).

2. The initial 1999 release of SSS data included about 5000 square degrees at the South Galactic Cap $(b<$ $-60^{\circ}$ ) with $B_{J}$ and $R$ photometry from the UKST plates as well as additional epoch measurements from UKST $I$ and ESO Schmidt $R$ plates. The proper motions given in the SSS catalogue were obtained from UKST $B_{J}$ and $R$ plates only, for all objects matched between the two passbands within a search radius of 3 arcsec. In order to find sources with larger proper motions, we selected unmatched objects which were picked up along a straight line on at least three plates with consecutive epochs (for more details see Lodieu et al. 2002). The proper motions were then refined by including all available positional information. Ultracool dwarf candidates were identified from these confirmed high proper motion objects by looking for the largest $B_{J}-R$ and $R-I$ indices.

3. The SSS UKST $B_{J}$ and $R$ all-southern-sky surveys were completed in 2001, with additional UKST $I$ survey and ESO $R$ survey data added subsequently. The catalogue 
photometric zero points were re-derived, resulting in significant changes in the $B_{J}-R$ and $R-I$ colours. Our revised search strategy then concentrated on the $R$ and $I$ plates alone, neglecting the blue $B_{J}$ plates which are much less sensitive to extremely red objects.

4. As an alternative strategy, we also looked for very red candidates among objects originally matched in all three passbands within a search radius of 6 arcsec and having a proper

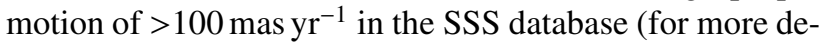
tails see Scholz \& Meusinger 2002). We subsequently reduced this limit to 50 mas $\mathrm{yr}^{-1}$ when the SSS proper motion determinations incorporated all plate measurements in all passbands, i.e., they were no longer based on the UKST $B_{J}$ and $R$ scans alone.

5. The SSS $I$ band survey was completed at the end of 2002 . As there is an overlap of 0.5 degrees on each side of an UKST plate with its neighbours, roughly one third of the southern sky SSS I band measurements had at least two different epochs. The epoch difference is non-uniform and generally only a few years, but nevertheless, these overlapping areas can be used to search for very high proper motion nearby brown dwarfs which may not show up on the $B_{J}$ and $R$ plates due to their extremely red colours. This new search method led to the discovery of nearest known brown dwarf, $\varepsilon$ Indi B (Scholz et al. 2003), subsequently resolved into a binary with spectral types T1 and T6 (McCaughrean et al. 2004).

For each of the five search strategies outlined above, we applied a magnitude limit at the faint end that ensured a sample of no more than a few thousand candidates. Source-by-source visual inspection then reduced the final sample to a few hundred objects for follow-up spectroscopic observations. Figure 1 shows the $I$ versus proper motion distribution for our current full sample of about 270 red proper motion objects, where filled symbols represent the objects described in the present paper. The effective faint magnitude limit of the combined sample of red proper motion objects is $I \sim 17.7 \mathrm{mag}$. Sources in Fig. 1 below this limit are ultracool L and $\mathrm{T}$ dwarfs culled from the literature which we subsequently found to be present in the SSS data but which were not picked up by our search strategies, mainly because they were below our applied magnitude limits.

Figure 1 also illustrates that our search strategy is sensitive to sources faint enough to be low-mass dwarfs even

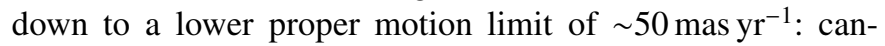
didates with proper motions this small were selected only if they also exhibited extremely red colours typical of late-M and early-L dwarfs in order to exclude more distant higher-mass sources. Our selected proper motion sample is also generally less complete at the lowest proper motions $\left(<300\right.$ mas yr $\left.^{-1}\right)$ due to the constraints set in the first search strategy. The subsample described in the present paper has a lower limit of about $80 \mathrm{mas} \mathrm{yr}^{-1}$. At the other extreme, objects with proper motions larger than $\sim 1 \operatorname{arcsec} \mathrm{yr}^{-1}$ were included as interesting candidates even if they had only moderately red colours, as these may be nearby mid-M dwarfs. The objects with the largest proper motions in Fig. 1, above the $1.14 \operatorname{arcsec}_{\mathrm{yr}^{-1}}$ upper limit in the present paper, were all newly discovered or recovered within the framework of our high proper motion survey. Some of these have already been published separately (Scholz et al. 2000, 2002, 2003, 2004b,c), whereas others are targets for upcoming spectroscopic follow-up. Five were previously discovered and classified by other surveys (Goldman et al. 1999; Martín et al. 1999; Delfosse et al. 2001; Lépine et al. 2003b; Hambly et al. 2004).

Figure 2 shows photographic optical and 2MASS nearinfrared colours as a function of spectral type for all objects spectroscopically classified in the present study, together with all M, L, and T dwarfs from the literature which could be identified in the SSS data. These detections of previously known objects include three further T dwarfs, SDSS 0423-04 (T0; Geballe et al. 2002), SDSS 1254-01 (T2; Leggett et al. 2000), and 2MASS 0559-14 (T5; Burgasser et al. 2000), in addition to $\varepsilon$ Indi B and ten L dwarfs already described by Scholz \& Meusinger (2002).

The optical-infrared colour indices of the $\mathrm{M}, \mathrm{L}$, and T dwarfs measured by the SSS (Fig. 2) increase monotonically with spectral types. Pure optical $R-I$ and infrared $J-K_{\mathrm{s}}$ colours saturate at about M8 and L5, respectively, but then turn bluer with later spectral type: the $R-I$ colour then starts becoming redder again at about L5. The smallest colour changes appear in $J-K_{\mathrm{s}}$, where a high photometric accuracy yields a small dispersion. Conversely, the largest effect is seen in $R-K_{\mathrm{s}}$, although its use as a selector would lead to the exclusion of some $\mathrm{L}$ and $\mathrm{T}$ dwarfs not visible shortward of the $I$ band. Finally, there are some clear outliers in the optical and optical-infrared colours labelled by name in Fig. 2: these objects will be discussed in Sect. 8.4.

The sample described in this paper contains a subset of 79 red high proper motion objects selected from our full candidate list (Table 1). As described in the following sections, we have obtained spectra with the VLT, NTT, and ESO 3.6-m at optical wavelengths for 58 of them and in the near-infrared for 27 , the latter including 19 objects already observed in the optical. VLT optical and near-infrared photometry was obtained for a subsample of objects as catalogued here, in addition to the $B_{J}, R$, and $I$ photographic plate measurements. Some comparison stars with known spectral types were also observed with identical setups: LHS 517 (M3.5), 2MASSW J0952-19 (M7.0), LP647-13 (M7.5), BRIB0021-0214 (M9.5), LP944-20 (M9.5), and Kelu 1 (L2.0). In addition, some red NLTT stars lacking spectral types were also observed. Finally, some of the objects in the sample were subsequently identified with previously known proper motion sources, including ESO 207-61, LHS 2555a, CE 303, LP 859-1, CE 352, [HB88] M18, and [HB88] M12.

\section{Observations and data reduction}

\subsection{VLT service mode observations}

An initial subsample of very red proper motion objects were observed photometrically and spectroscopically on the ESO VLT UT1 in service mode in 1999 (P63.L-0634) and in 2000 (P65.L-0689) in the optical with the Focal Reducer/Low Dispersion Spectrograph FORS 1 and in the nearinfrared with the Infrared Spectrometer And Array Camera 
(ISAAC). The observations were conducted in dark and grey time in the optical and near-infrared, respectively, and under good seeing conditions $(\leq 0.8$ arcsec) as requested.

\subsubsection{VLT/FORS1 imaging}

FORS1 employs a $2048 \times 2048$ pixel thinned TK CCD detector: the image scale of 0.20 arcsec yields a field-of-view of $6.8 \times 6.8 \mathrm{arcmin}$. A series of three dithered positions was exposed for 10 and $5 \mathrm{~s}$ each in each of the Bessell $R$ and $I$ filters, respectively, in order to derive more accurate magnitudes and colours than were available from the plate material. Standard stars were observed each night in order to calibrate the photometry. Data reduction was standard, with each frame biassubtracted and flat-fielded prior to aperture photometry. Table 2 lists the mean magnitude calculated from three independent measurements, the exposure times, and the specific observing dates for 32 sources in the initial subsample.

\subsubsection{VLT/FORS1 spectroscopy}

Of the 32 sources observed photometrically with FORS1, a subset of 22 were followed-up with low-resolution FORS1 spectroscopy $(R \sim 600)$ with grism GRIS_300I+11 spanning 6000-11000 $\AA$, apart from APMPM J2036-4936 and LP 859-1 which were observed with grism GRIS_150I+17 covering 3500-11000 ̊̊. Total exposure times were set according to the brightness of the object as listed in Table 3. Data reduction involved subtracting an averaged dark frame and dividing by a dome flat field. Wavelength calibration was carried out using $\mathrm{He}$ and $\mathrm{Ar}$ arc lamp lines covering the whole wavelength range. The data were flux calibrated using an averaged sensitivity function created from a spectrophotometric standard star observed on the same night. The spectra shown in Figs. 3 and 4 have been normalised at $7500 \AA$ for consistency with existing spectral classification schemes (Kirkpatrick et al. 1999; Martín et al. 1999). No telluric correction was applied to the optical spectra.

\subsubsection{VLT/ISAAC imaging}

All but two (LP 314-67 and LDS 4980A) of the sources imaged with FORS1 were then imaged with ISAAC to obtain near-infrared photometry. ISAAC employs a HAWAII $1024 \times$ 1024 pixel $\mathrm{HgCdTe}$ array covering the $1-2.5 \mu$ m wavelength range with a pixel size of 0.147 arcsec, yielding a field of view of $2.5 \times 2.5 \mathrm{arcmin}$. A series of five dithered positions was observed in each of three broad-band filters $\left(J_{\mathrm{s}}, H, K_{\mathrm{s}}\right)$ : for each position in each filter, a flat-field correction was applied and an average of the other four frames was used to subtract the sky flux. Aperture photometry was carried out for each individual filter. The magnitudes listed in Table 2 correspond to the average of all five measurements in each filter. It should be noted that the ISAAC $J_{\mathrm{s}}$ filter is non-standard, thus potentially leading to problems when comparing with existing calibrations. However, although it was not available when we began our observational program, the 2MASS Second Incremental Data Release in March 2000 provided standard $J$ magnitudes for our entire sample, and it is these magnitudes which are in Table 1 and are used for all calculations made in this paper.

\subsubsection{VLT/ISAAC spectroscopy}

Low-resolution $(R \sim 600)$ spectroscopy spanning $1-2.5 \mu \mathrm{m}$ was obtained with ISAAC for 6 objects (see Table 3 ) including Kelu 1 (Ruiz et al. 1997), which was observed as a template. A slit width of 1 arcsec was employed throughout and three gratings were used to cover the spectral ranges $1.10-1.39 \mu \mathrm{m}$, $1.42-1.83 \mu \mathrm{m}$, and $1.84-2.56 \mu \mathrm{m}$. The sources were measured at three or more positions along the slit to permit removal of the sky spectrum. Featureless standards (typically F and G stars) were measured within one degree on the sky of each source and close in time in order to remove telluric absorption. Each individual frame was flat-fielded, sky-subtracted, and a onedimensional spectrum extracted. These spectra were then divided by the standard, multiplied by an appropriate spectral template smoothed to our resolution, and then co-added to increase the signal-to-noise. The spectra as shown in Fig. 6 were normalised at $1.28 \mu \mathrm{m}$.

\subsection{ESO 3.6-m/EFOSC2 spectroscopy}

A second subsample of 47 very red, high proper motion sources was observed in the optical with EFOSC2 on the ESO 3.6-m telescope on La Silla on 22-23 November 2001 and 5-8 December 2002. The weather during the 2001 run was photometric, with seeing $\sim 0.6-0.8$ arcsec FWHM; the conditions during the 2002 run were poorer, with some observations affected by thin clouds and seeing of $\sim 1.0-1.5$ arcsec FWHM.

EFOSC2 uses a $2048 \times 2048$ pixel Loral/Lesser CCD with a pixel size of 0.157 arcsec, yielding a useful field of view of $5.2 \times 5.2$ arcmin. Spectroscopy was obtained for most of the objects just once, but additional data were taken for potentially interesting candidates or targets with poorer signal-tonoise. In these latter cases, the sources were observed at two or three positions shifted along the slit by 100 pixels. The details for the observations for each source are given in Table 4 . The data were reduced in a similar fashion to that described for the VLT/FORS1 spectroscopy, with the addition that an internal quartz flat field taken immediately after the first exposure in each series was used to remove fringing above $8000 \AA$. The spectra were normalised at $7500 \AA$ and are displayed in Figs. 5-11. No telluric absorption correction was applied to the spectra.

\subsection{NTT/SOFI spectroscopy}

Additional near-infrared $(0.95-2.5 \mu \mathrm{m})$ spectroscopy was obtained using SOFI on the ESO NTT on La Silla on 24-25 November 2001 for sources identified for followup from the EFOSC2 optical spectroscopy and for sources with interesting near-infrared colours in the 2MASS database. Both nights were photometric with seeing varying between $0.8-1.0$ arcsec FWHM.

SOFI employs a HAWAII $1024 \times 1024$ pixel HgCdTe array (Moorwood \& Spyromilio 1999) with a pixel size of 0.294 arcsec delivered by the Large Field Objective used 

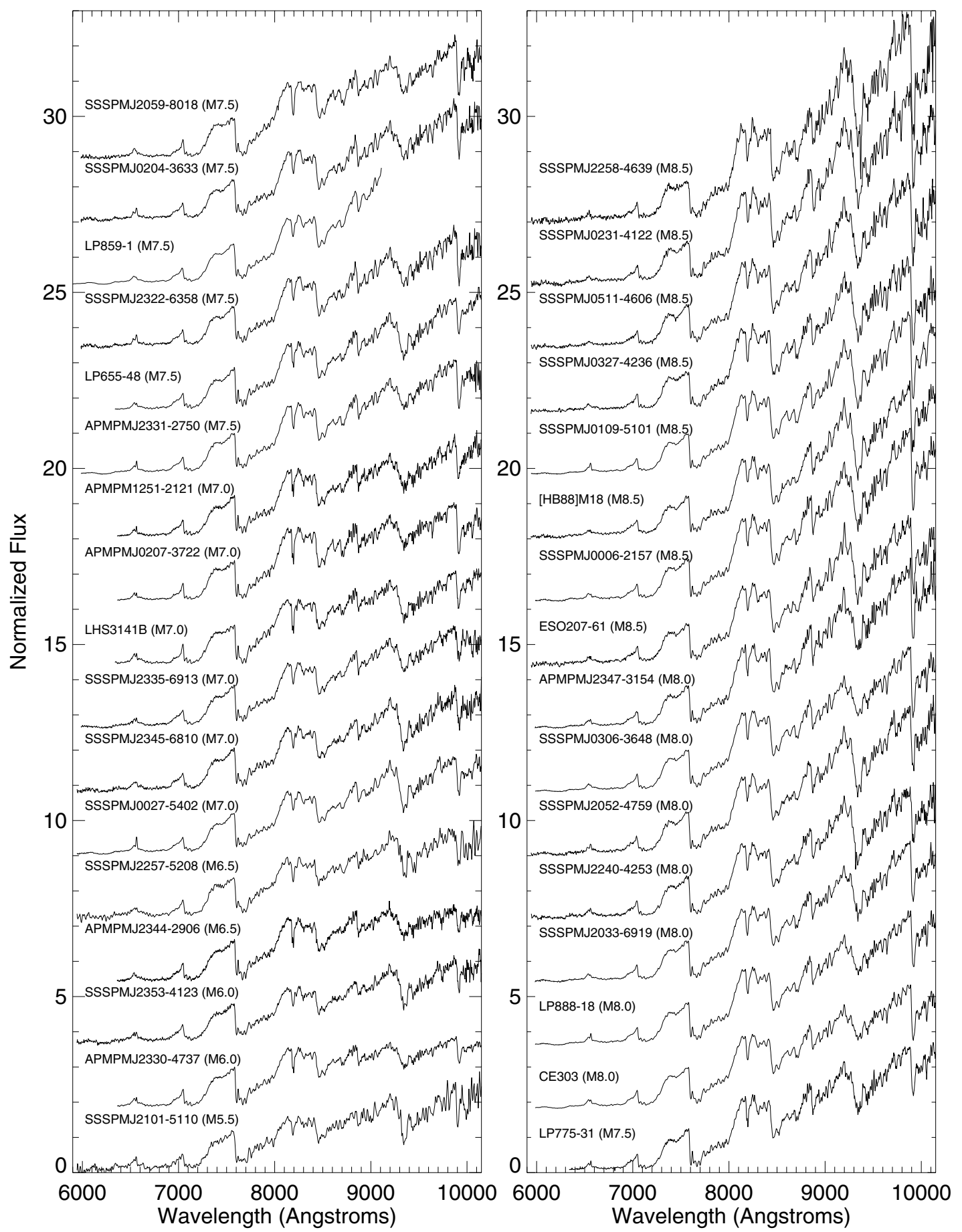

Fig. 3. Optical spectra of sources with spectral types between M5.5 and M8, assigned according to the Kirkpatrick et al. (1999) and Martín et al. (1999) classification schemes. The uncertainty on the spectral type is typically half a subclass. An arbitrary constant has been added to separate the spectra.

for spectroscopy. A 1 arcsec slit was used yielding $R \sim 600$ for both blue $(0.95-1.64 \mu \mathrm{m})$ and red $(1.53-2.52 \mu \mathrm{m})$ gratings. Featureless standards (typically F5-F7) were measured within one degree on the sky of each source to remove telluric absorption and flux calibrate. Each source was observed at three positions along the slit to facilitate sky subtraction. The data were reduced in the same way as described in Sect. 3.1.4. The spectra were again normalised at $1.28 \mu \mathrm{m}$ and are shown in Fig. 6.

\section{Spectroscopic classification of $M$ and $L$ dwarfs}

\subsection{Optical classification}

Two independent classification schemes for $\mathrm{M}$ and $\mathrm{L}$ dwarfs based on their optical spectra have recently been defined by Kirkpatrick et al. (1999) and Martín et al. (1999). The former scheme is based on spectral ratios in different regions of interest and on spectrum comparisons, while the second scheme is primarily based on a pseudo-continuum ratio known 


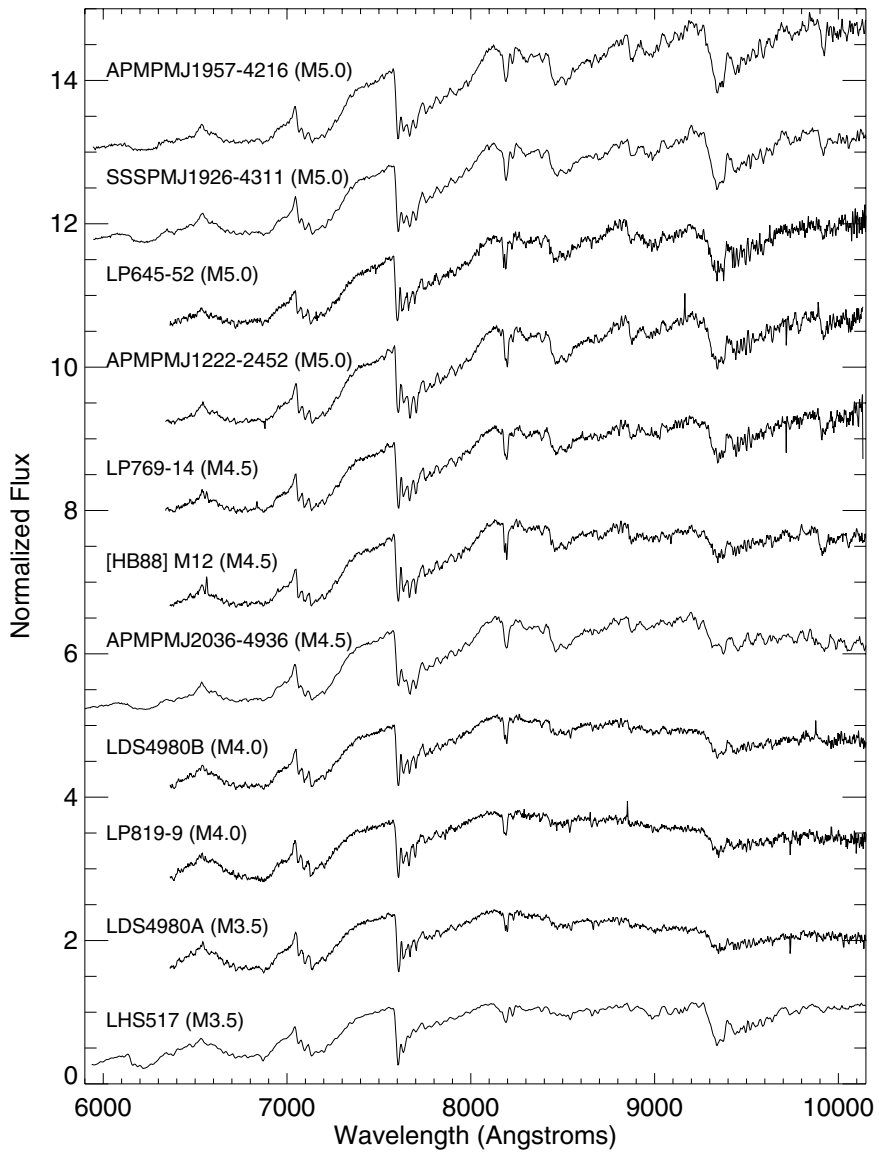

Fig. 4. Optical spectra of objects earlier than M5.5 according to the Kirkpatrick et al. (1999) and Martín et al. (1999) classification schemes. The uncertainty on the spectral type is typically half a subclass. An arbitrary constant has been added to separate the spectra.

as PC3. In this paper, we have adopted classifications based on a hybrid of the most reliable indices for late $\mathrm{M}$ and early L dwarfs, namely TiO5 (Reid et al. 1995; Cruz \& Reid 2002), VO-a (Kirkpatrick et al. 1999; Cruz \& Reid 2002), and PC3 (Martín et al. 1999), as well the direct comparison with objects of known spectral type from Kirkpatrick et al. (1999), Gizis et al. (2000), and Reid et al. (1999) which we observed with the same instrument setups as our candidates. We also compared our spectra with templates available from other groups, including those of Neill Reid ${ }^{1}$.

Table 5 lists the values and the derived spectral types measured from the TiO5, VO-a, and PC3 indices, the spectral type determined by direct visual comparison, and the spectral type finally adopted for each source. The adopted spectral type was arrived at as follows. First, the mean value of the three independent indices was calculated. This value was adopted unless it was seen to be in gross disagreement with that determined from the template comparison, in which case the template comparison value was preferred. Although most spectral type determinations were indeed in good agreement, discrepancies were seen for a small number of objects, namely APMPM J2331-2750, SSSPM J2322-6358, SSSPM J2059-8018, SSSPM J2353-4123, SSSPM J0327-4236, and

\footnotetext{
${ }^{1}$ http://dept.physics. upenn. edu/ inr/ultracool .html
}

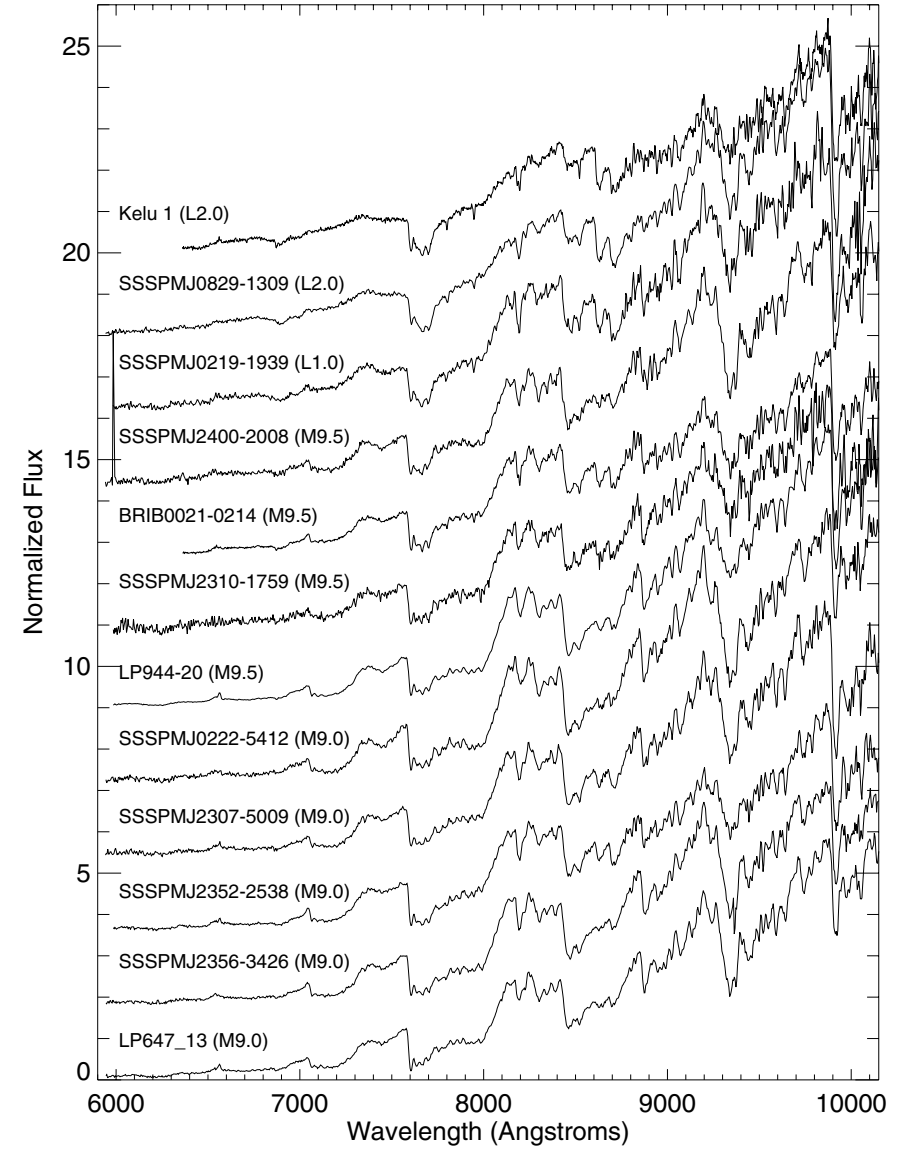

Fig. 5. Optical spectra of the latest-type (M9 to L2) objects, following the Kirkpatrick et al. (1999) and Martín et al. (1999) classification schemes. The uncertainty on the spectral type is typically half a subclass. An arbitrary constant has been added to separate the spectra.

SSSPM J2258-4639. For each of these, the spectral type obtained from comparison with templates was adopted.

\subsection{Near-infrared classification}

A total of 27 objects were observed spectroscopically in the near-infrared. We have used a variety of different near-infrared classification schemes which were designed to provide a best match to the optical classifications for M and L dwarfs (Reid et al. 2001; Tokunaga \& Kobayashi 1999; Martín 2000). The reader is referred to those papers for more details on each scheme.

Table 6 lists the numerical indices measured in each scheme, the corresponding spectral type, and the combined spectral type obtained by taking their mean for each source. Since 19 of these sources also have optical spectra, we are able to compare the optical and near-infrared classification schemes (Fig. 10). There appears to be a systematic shift between the schemes, with generally later spectral types (by roughly one subclass) being assigned in the near-infrared. This discrepancy and the relatively large scatter tends to suggest that the nearinfrared schemes are less reliable that their optical counterparts, somewhat at odds with the findings of other studies (see, e.g., Reid et al. 2001). 

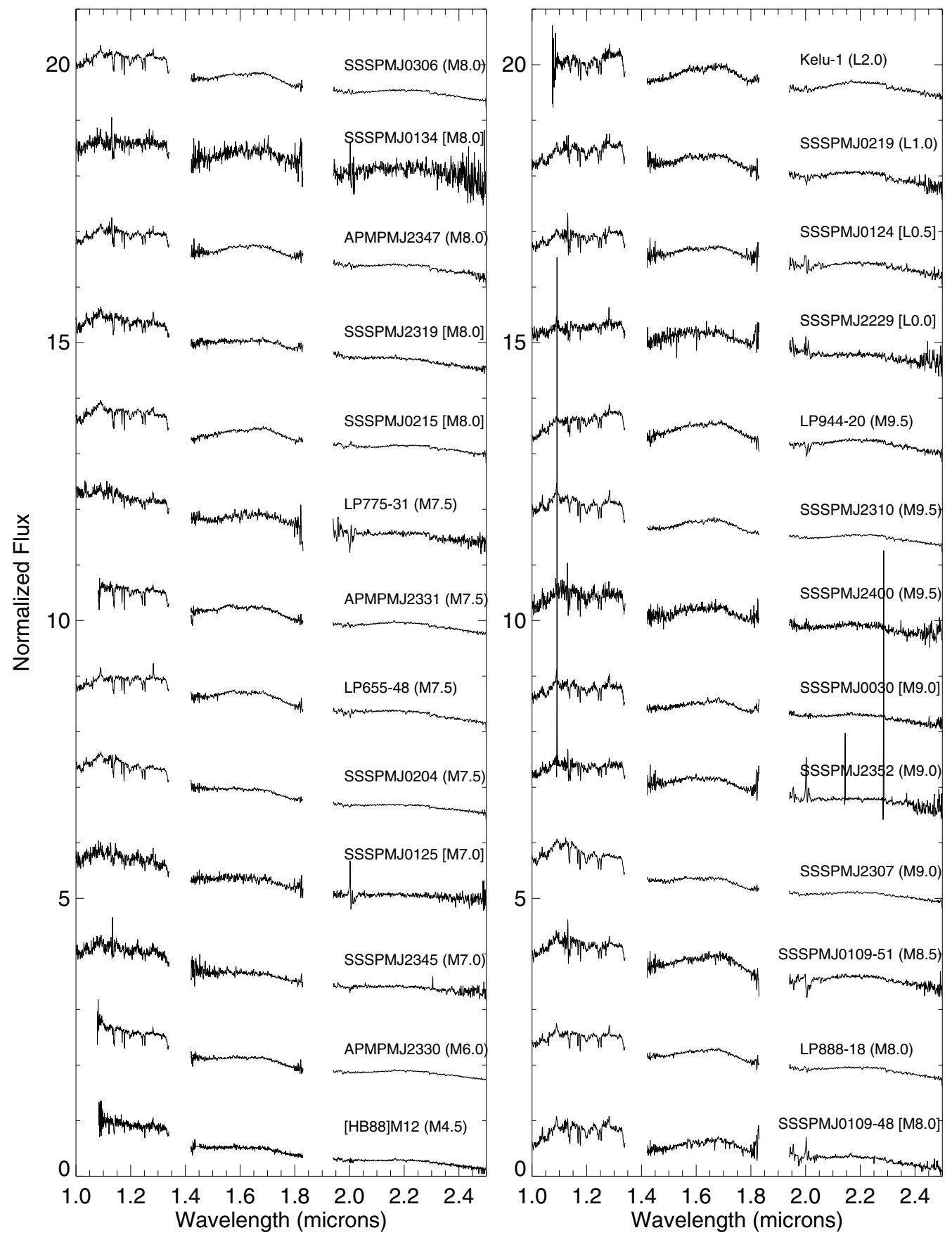

Fig. 6. Near-infrared spectra of a subsample of the objects. Objects with spectral types in square brackets have been classified based on their near-infrared spectra alone, while those listed in parentheses are derived from the optical spectrum for each source. An arbitrary constant has been added to separate the spectra.

As a consequence, we have adopted spectral types for the sources with near-infrared spectra in the following manner. For the 19 sources with optical spectra, we have simply taken the optical classification. For the remaining 8 sources with nearinfrared spectra only, we have ignored the numerical indices and have instead relied on direct comparison of the spectra with sources with known spectral types which we observed with the same setups (SSSPM J0306-3648, LP 944-20, Kelu 1), or templates made available by Neill Reid (see above) and Sandy
Leggett ${ }^{2}$. The various computed indices, the associated spectral types, and the finally adopted spectral types are listed in Table 6 . We calculated a formal uncertainty of half a subclass in the near-infrared classification. However, due to the systematic observed discrepancy between the optical and nearinfrared spectral types of about one subclass, we adopt an error of one subclass on the near-infrared spectral types to be on the cautious side.

\footnotetext{
${ }^{2}$ http://www.jach.hawaii.edu/ skl/data.html
} 


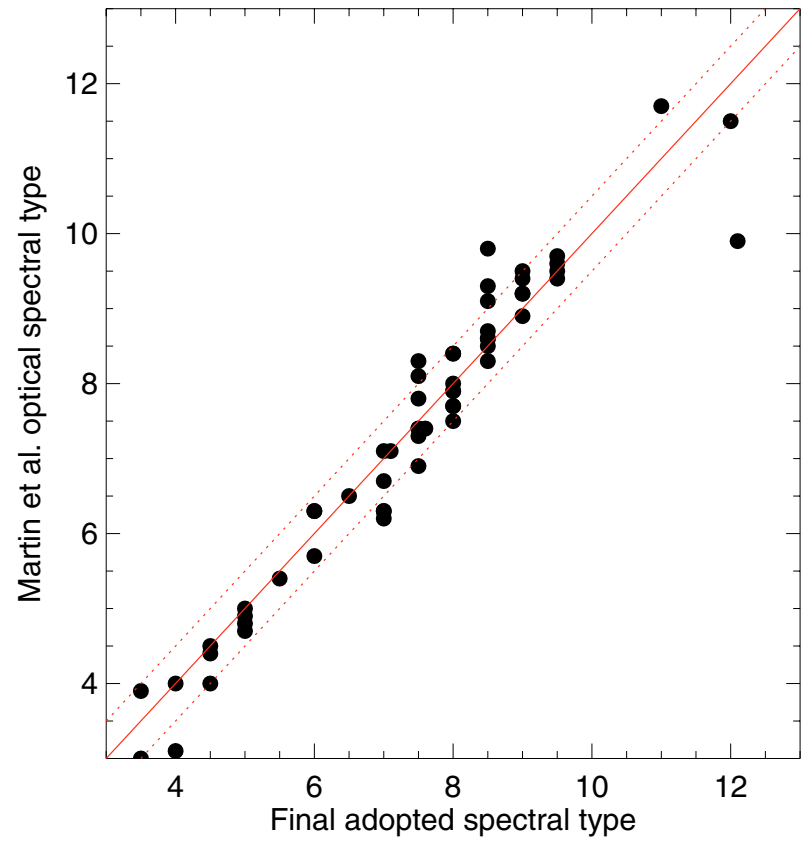

Fig. 7. The final optical spectral types $(4 \equiv M 4, \ldots, 12 \equiv \mathrm{L} 2)$ adopted for each source in this paper vs. spectral types derived from the classification scheme of Martín et al. (1999). The solid line represents equal spectral types, while the dashed lines show differences of half a spectral type. Some sources have been shifted slightly on the $x$-axis for clarity.

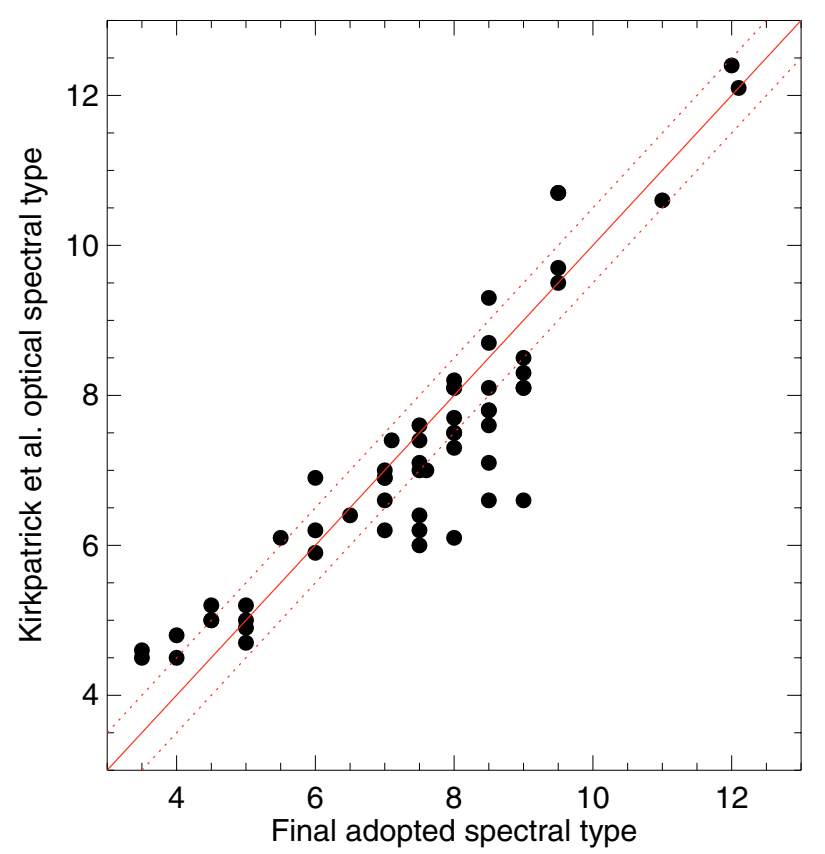

Fig. 8. The final optical spectral types $(4 \equiv M 4, \ldots, 12 \equiv \mathrm{L} 2)$ adopted for each source in this paper vs. spectral types derived from the classification scheme of Kirkpatrick et al. (1999). Some sources have been shifted slightly on the $x$-axis for clarity.

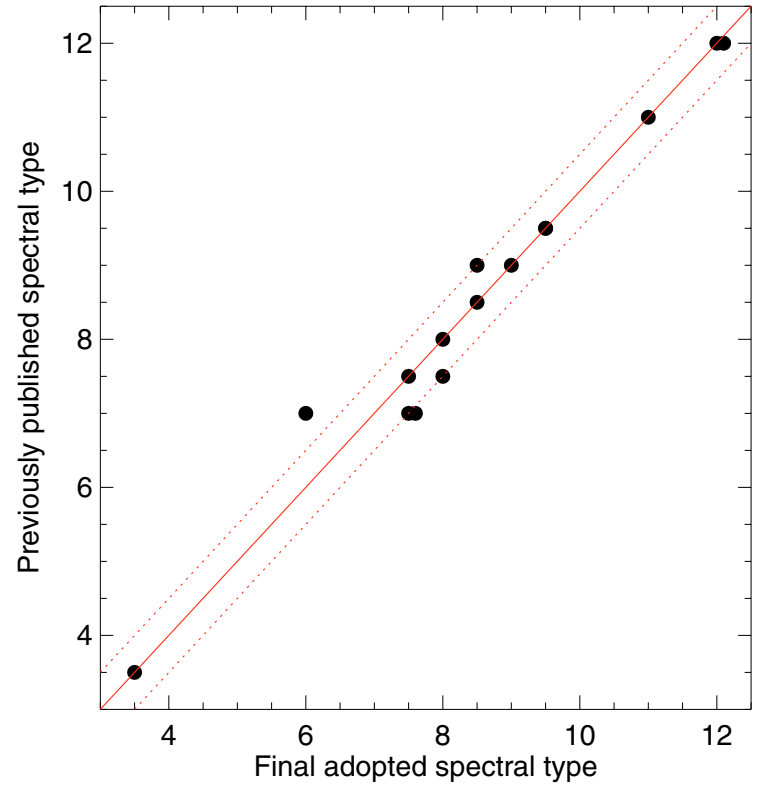

Fig. 9. The adopted optical spectral type vs. previous classifications found in the literature $(4 \equiv \mathrm{M} 4, \ldots, 12 \equiv \mathrm{L} 2)$. The latter were obtained from: Kirkpatrick et al. (2000): BRI B0021-0214 (M9.5); Ruiz et al. 1997: Kelu 1 (L2.0); Tinney 1998: LP 944-20 (M9.5); Reid et al. (2002b): LP 859-1 (M7.5); Gizis et al. (2000): 2MASSW J0952-19 (M7.0); Cruz \& Reid (2002): LP 647-13 (M9.0); Ianna \& Fredrick (1995): ESO 207-61 (M9.0); Scholz \& Meusinger (2002): SSSPM J0829-1309 (L2.0); Hawkins \& Bessell (1988): [HB88] M18 (M8.5); Hawley et al. (1996): LHS 517 (M3.5); Cruz et al. (2003): SSSPMJ0219-1939 (L1.0), LP 888-18 (M7.5), LP775-31 (M7.0), LP 655-48 (M7.0), and CE 303 (M8.0). 2MASSW J0952-19 is the only object with more than half a spectral type difference in its classification. Some sources have been shifted slightly on the $x$-axis for clarity.

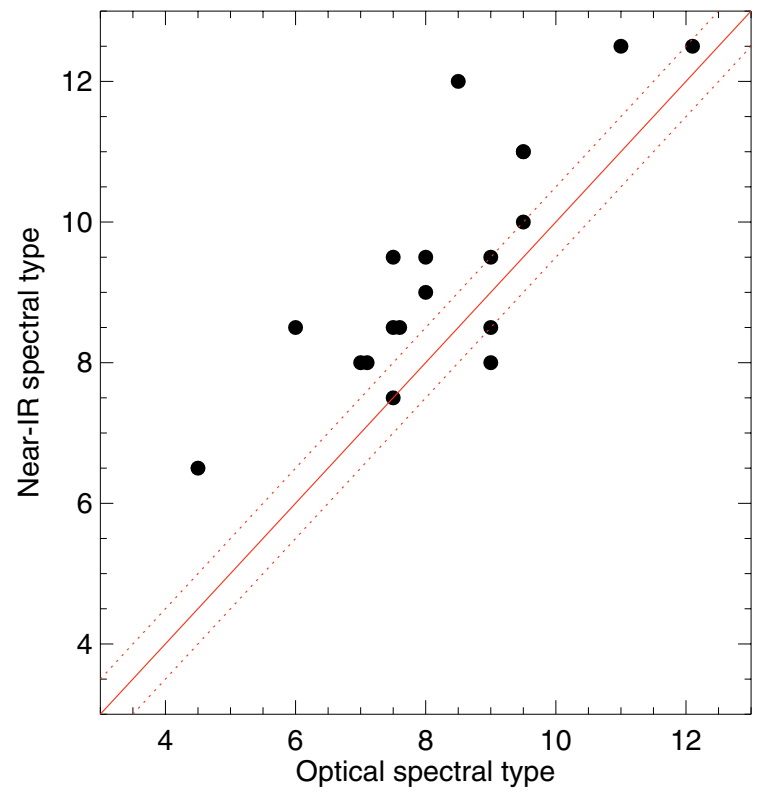

Fig. 10. Spectral types determined from our optical spectra vs. those obtained from our near-infrared spectra by taking the mean of the numerical indices of Reid et al. (2001), Tokunaga \& Kobayashi (1999), and Martín (2000) $(4 \equiv M 4, \ldots, 12 \equiv \mathrm{L} 2)$. Some sources have been shifted slightly on the $x$-axis for clarity. 
Table 5. Results from the optical spectra for all $\mathrm{M}$ and $\mathrm{L}$ dwarfs in our sample. The measured $\mathrm{H} \alpha$ index and equivalent width as discussed in the text are listed first. Then optical indices (TiO5, VO-a, and PC3) and spectral types in the schemes of Kirkpatrick et al. (1999) and Martín et al. (1999) are shown. SpT (TiO5), SpT (VO-a), and SpT (PC3) show the numerical values and corresponding spectral type for the three indices, while SpT (Comp) shows the spectral type assigned via direct comparison with template objects. The final, adopted spectral type corresponds to the mean value of the three spectral indices rounded to the nearest half spectral type or to that obtained from direct comparison as discussed in the text. The typical uncertainty of the adopted spectral type is half a subclass. The distance shown is derived from the relation between the absolute $M_{J}$ magnitude and the spectral type presented in Dahn et al. (2002); $v_{\mathrm{t}}$ is the resulting tangential velocity.

\begin{tabular}{|c|c|c|c|c|c|c|c|c|c|c|}
\hline $\mathrm{RN}$ & Name & $\begin{array}{c}\mathrm{H} \alpha \\
\text { index }\end{array}$ & $\begin{array}{r}\mathrm{H} \alpha \\
\mathrm{EW}\end{array}$ & $\begin{array}{l}\text { SpT } \\
\text { TiO5 }\end{array}$ & $\begin{array}{c}\text { SpT } \\
\text { VO-a }\end{array}$ & $\begin{array}{l}\mathrm{SpT} \\
\mathrm{PC} 3\end{array}$ & $\begin{array}{c}\text { SpT } \\
\text { Comp }\end{array}$ & $\begin{array}{l}\text { SpT } \\
\text { final }\end{array}$ & $\begin{array}{c}\text { distance } \\
{[\mathrm{pc}]}\end{array}$ & $\begin{array}{c}v_{\mathrm{t}} \\
{\left[\mathrm{km} \mathrm{s}^{-1}\right]}\end{array}$ \\
\hline 01 & SSSPM J0006-2157 & 1.013 & 1.9 & 0.275 (M7.8) & $2.290(\mathrm{M} 7.8)$ & 1.982 (M8.6) & M8.5 & M8.5 & $25.0 \pm 3.0$ & 87 \\
\hline 02 & BRI B0021-0214 & 0.938 & 0.0 & 0.670 (L0.0) & 2.275 (L0.7) & 2.344 (M9.7) & M9.5 & M9.5 & $11.9 \pm 1.4$ & 9 \\
\hline 03 & SSSPM J0027-5402 & 1.947 & 14.5 & 0.278 (M7.8) & 2.213 (M7.0) & 1.500 (M6.3) & M7.0 & M7.0 & $20.8 \pm 2.5$ & 41 \\
\hline 05 & LP 645-52_1 & 0.920 & 0.2 & 0.314 (M4.8) & 1.996 (M4.7) & 1.276 (M5.0) & M5.0 & M5.0 & $100.5 \pm 12.1$ & 209 \\
\hline 05 & LP 645-52_2 & 0.930 & 0.2 & 0.310 (M4.9) & 2.012 (M4.9) & 1.228 (M4.6) & M5.0 & M5.0 & $100.5 \pm 12.1$ & 209 \\
\hline 07 & SSSPM J0109-5101 & 1.320 & 13.3 & 0.328 (M8.1) & 2.293 (M7.8) & 1.996 (M8.6) & M8.5 & M8.5 & $15.5 \pm 1.9$ & 17 \\
\hline 09 & LP647-13 & 1.310 & -7.9 & 0.56 (M9.4) & 2.360 (M8.5) & 2.080 (M8.9) & M9.0 & M9.0 & $11.2 \pm 1.3$ & 19 \\
\hline 13 & LP 769-14 & 1.136 & 2.5 & 0.335 (M4.6) & 2.023 (M5.0) & 1.204 (M4.5) & M4.5 & M4.5 & $159.2 \pm 19.1$ & 142 \\
\hline 14 & SSSPM J0204-3633 & 1.529 & 10.8 & 0.252 (M7.6) & $2.254(\mathrm{M} 7.4)$ & 1.701 (M7.4) & M7.5 & M7.5 & $29.3 \pm 3.5$ & 31 \\
\hline 16 & APMPM J0207-3722 & 1.507 & 6.7 & $0.210(\mathrm{M} 7.4)$ & $2.257(\mathrm{M} 7.4)$ & $1.637(\mathrm{M} 7.1)$ & M7.0 & M7.0 & $21.6 \pm 2.6$ & 46 \\
\hline 18 & SSSPM J0219-1939 & 0.935 & 0.0 & $0.951(\mathrm{~L} 1.6)$ & $2.289(\mathrm{~L} 0.6)$ & $3.016(\mathrm{~L} 1.7)$ & L0.5 & $\mathrm{L} 1.0$ & $24.9 \pm 3.0$ & 31 \\
\hline 19 & SSSPM J0222-5412 & 1.059 & 0.0 & 0.433 (M8.7) & 2.643 (M8.1) & 2.163 (M9.2) & M9.0 & M9.0 & $30.9 \pm 3.7$ & 16 \\
\hline 20 & SSSPM J0231-4122 & 0.779 & 0.0 & 0.414 (M8.6) & 2.267 (M7.6) & 2.194 (M9.3) & M8.5 & M8.5 & $32.7 \pm 3.9$ & 51 \\
\hline 23 & SSSPM J0306-3648 & 0.978 & 0.5 & $0.224(\mathrm{M} 7.5)$ & $2.241(\mathrm{M} 7.3)$ & 1.924 (M8.4) & M8.0 & M8.0 & $13.1 \pm 1.6$ & 43 \\
\hline 24 & SSSPM J0327-4236 & 0.925 & 0.0 & $0.251(\mathrm{M} 7.6)$ & $2.226(\mathrm{M} 7.1)$ & 2.133 (M9.1) & M8.5 & M8.5 & $38.8 \pm 4.7$ & 51 \\
\hline 26 & LP $888-18$ & 1.544 & 11.0 & 0.264 (M7.7) & $2.259(\mathrm{M} 7.5)$ & 1.817 (M7.9) & M8.0 & M8.0 & $11.2 \pm 1.3$ & 21 \\
\hline 27 & LP 944-20 & 1.001 & 0.0 & 0.521 (M9.2) & 2.455 (M9.5) & 2.258 (M9.4) & M9.5 & M9.5 & $6.6 \pm 0.8$ & 13 \\
\hline 28 & LP775-31 & 1.183 & 3.7 & 0.257 (M7.7) & $2.219(\mathrm{M} 7.0)$ & 1.737 (M7.6) & M7.5 & M7.5 & $7.8 \pm 0.9$ & 13 \\
\hline 29 & LP655-48 & 1.480 & 10.0 & $0.282(\mathrm{M} 7.8)$ & $2.213(\mathrm{M} 7.0)$ & $1.666(\mathrm{M} 7.2)$ & M7.5 & M7.5 & $8.8 \pm 1.1$ & 15 \\
\hline 31 & SSSPM J0511-4606 & 0.796 & 0.0 & $0.311(\mathrm{M} 8.0)$ & 2.477 (M9.3) & 2.005 (M8.7) & M8.5 & M8.5 & $33.3 \pm 4.0$ & 21 \\
\hline 33 & ESO 207-61 & 1.174 & 2.8 & $0.311(\mathrm{M} 8.0)$ & $2.321(\mathrm{M} 8.1)$ & 1.969 (M8.5) & M8.5 & M8.5 & $24.6 \pm 2.9$ & 47 \\
\hline 34 & SSSPM J0829-1309 & 0.996 & 0.0 & 1.008 (L1.9) & $2.033(\mathrm{~L} 2.4)$ & $2.753(\mathrm{~L} 1.5)$ & L2.0 & $\mathrm{L} 2.0$ & $11.6 \pm 1.4$ & 33 \\
\hline 36 & 2MASSW J0952-19 & 1.575 & 11.3 & 0.240 (M5.6) & 2.106 (M5.9) & 1.489 (M6.3) & M6.0 & M6.0 & $21.0 \pm 2.5$ & 13 \\
\hline 39 & APMPM J1222-2452 & 0.867 & 0.0 & 0.287 (M5.1) & 2.041 (M5.2) & 1.253 (M4.8) & M5.0 & M5.0 & $129.4 \pm 15.5$ & 279 \\
\hline 41 & APMPM J1251-2121 & 1.109 & 2.6 & 0.238 (M7.6) & $2.272(\mathrm{M} 7.6)$ & 1.614 (M6.9) & M7.5 & M7.5 & $11.1 \pm 1.3$ & 29 \\
\hline 42 & Kelu 1_1 & 0.933 & 0.0 & 0.965 (L1.7) & 1.983 (L2.8) & 2.128 (L0.9) & L2.0 & $\mathrm{L} 2.0$ & $15.4 \pm 1.8$ & 24 \\
\hline 42 & Kelu 1_2 & 1.163 & 0.0 & 1.009 (L1.9) & 2.087 (L2.1) & $2.471(\mathrm{~L} 1.2)$ & L2.0 & $\mathrm{L} 2.0$ & $15.4 \pm 1.8$ & 24 \\
\hline 43 & CE 303 & 1.456 & 6.8 & $0.258(\mathrm{M} 7.7)$ & $2.322(\mathrm{M} 8.1)$ & $1.771(\mathrm{M} 7.7)$ & M8.0 & M8.0 & $13.6 \pm 1.6$ & 24 \\
\hline 45 & LP 859-1 & 0.974 & 0.2 & $-(-)$ & $2.228(\mathrm{M} 7.1)$ & 1.697 (M7.4) & M7.5 & M7.5 & $16.4 \pm 2.0$ & 27 \\
\hline 46 & LHS 3141B & 1.245 & 4.8 & 0.115 (M6.9) & 2.059 (M6.9) & $1.645(\mathrm{M} 7.1)$ & M7.0 & M7.0 & $37.0 \pm 4.4$ & 101 \\
\hline 47 & SSSPM J1926-4311 & 0.912 & 0.0 & 0.303 (M4.9) & 2.018 (M4.9) & $1.230(\mathrm{M} 4.7)$ & M5.0 & M5.0 & $43.0 \pm 5.2$ & 222 \\
\hline 48 & APMPM J1957-4216 & 0.966 & 0.0 & 0.264 (M5.4) & 2.024 (M5.0) & 1.273 (M4.9) & M5.0 & M5.0 & $54.4 \pm 6.5$ & 266 \\
\hline 50 & SSSPM J2033-6919 & 0.983 & 1.0 & $0.214(\mathrm{M} 7.4)$ & 2.281 (M7.7) & 1.803 (M7.9) & M8.0 & M8.0 & $32.1 \pm 3.9$ & 78 \\
\hline 51 & APMPM J2036-4936 & 0.859 & 0.0 & $0.292(\mathrm{M} 5.0)$ & 2.020 (M5.0) & $1.140(\mathrm{M} 4.0)$ & M4.0 & M4.5 & $165.8 \pm 19.9$ & 335 \\
\hline 52 & SSSPM J2052-4759 & 1.475 & 12.1 & 0.303 (M7.9) & $2.333(\mathrm{M} 8.2)$ & 1.847 (M8.0) & M8.0 & M8.0 & $23.2 \pm 2.8$ & 48 \\
\hline 53 & SSSPM J2059-8018 & 0.869 & 0.2 & 0.134 (M6.8) & 2.123 (M6.0) & 1.904 (M8.3) & M7.5 & M7.5 & $46.6 \pm 5.6$ & 81 \\
\hline 54 & SSSPM J2101-5110 & 1.212 & 7.1 & 0.299 (M5.0) & 2.129 (M6.1) & 1.341 (M5.4) & M6.0 & M5.5 & $107.7 \pm 12.9$ & 72 \\
\hline 55 & [HB88] M18 & 1.044 & 0.0 & 0.364 (M8.3) & 2.381 (M8.7) & 1.913 (M8.3) & M8.5 & M8.5 & $26.9 \pm 3.2$ & 78 \\
\hline 56 & [HB88] M12 & 1.556 & 6.6 & 0.279 (M5.2) & 2.044 (M5.2) & 1.190 (M4.4) & M4.5 & M4.5 & $153.0 \pm 18.4$ & 62 \\
\hline 57 & LP 819-9 & 0.938 & 0.0 & 0.384 (M4.1) & 2.006 (M4.8) & 1.134 (M4.0) & M4.0 & M4.0 & $199.8 \pm 24.0$ & 269 \\
\hline 58 & LHS 517 & 0.956 & 0.0 & 0.506 (M2.7) & 1.975 (M4.5) & 1.004 (M3.0) & M3.5 & M3.5 & $5.1 \pm 0.6$ & 27 \\
\hline 60 & LDS4980 B & 0.992 & 0.6 & 0.377 (M4.1) & 1.984 (M4.6) & 1.124 (M3.9) & M4.0 & M4.0 & $195.6 \pm 23.5$ & 220 \\
\hline
\end{tabular}


Table 5. continued.

\begin{tabular}{|c|c|c|c|c|c|c|c|c|c|c|}
\hline $\mathrm{RN}$ & Name & $\begin{array}{c}\mathrm{H} \alpha \\
\text { index }\end{array}$ & $\begin{array}{l}\mathrm{H} \alpha \\
\mathrm{EW}\end{array}$ & $\begin{array}{l}\text { SpT } \\
\text { TiO5 }\end{array}$ & $\begin{array}{l}\text { SpT } \\
\text { VO-a }\end{array}$ & $\begin{array}{l}\text { SpT } \\
\text { PC3 }\end{array}$ & $\begin{array}{c}\mathrm{SpT} \\
\text { Comp }\end{array}$ & $\begin{array}{l}\text { SpT } \\
\text { final }\end{array}$ & $\begin{array}{c}\text { distance } \\
{[\mathrm{pc}]}\end{array}$ & $\begin{array}{c}v_{\mathrm{t}} \\
{\left[\mathrm{km} \mathrm{s}^{-1}\right]}\end{array}$ \\
\hline 61 & LDS4980 A & 0.927 & 0.0 & 0.443 (M3.4) & 1.978 (M4.5) & 1.017 (M3.1) & M3.5 & M3.5 & $229.2 \pm 27.5$ & 255 \\
\hline 62 & SSSPM J2240-4253 & 1.238 & 7.1 & 0.254 (M7.7) & $2.257(\mathrm{M} 7.5)$ & 1.774 (M7.7) & M8.0 & M8.0 & $33.9 \pm 4.1$ & 87 \\
\hline 63 & SSSPM J2257-5208 & 1.006 & 2.6 & 0.196 (M6.1) & 2.139 (M6.2) & 1.472 (M6.2) & M7.0 & M7.0 & $67.9 \pm 8.1$ & 41 \\
\hline 64 & SSSPM J2258-4639 & 0.833 & 0.0 & $0.231(\mathrm{M} 7.5)$ & 2.181 (M6.6) & 2.430 (M9.8) & M8.5 & M8.5 & $29.3 \pm 3.5$ & 38 \\
\hline 65 & SSSPM J2307-5009 & 1.318 & 2.0 & 0.513 (M9.1) & 2.334 (M8.3) & 2.265 (M9.5) & M8.5 & M9.0 & $24.4 \pm 2.9$ & 53 \\
\hline 66 & SSSPM J2310-1759 & 1.550 & 0.0 & 0.520 (M9.2) & 2.280 (L0.7) & 2.273 (M9.5) & M9.5 & M9.5 & $35.7 \pm 4.3$ & 48 \\
\hline 68 & SSSPM J2322-6358 & 1.600 & 11.2 & 0.244 (M5.6) & 2.161 (M6.4) & 1.677 (M7.3) & M7.5 & M7.5 & $46.2 \pm 5.5$ & 27 \\
\hline 69 & APMPM J2330-4737 & 1.292 & 5.6 & 0.204 (M6.0) & 2.142 (M6.2) & 1.495 (M6.3) & M6.0 & M6.0 & $15.8 \pm 1.9$ & 86 \\
\hline 70 & APMPM J2331-2750 & 1.297 & 5.1 & 0.181 (M6.2) & 2.137 (M6.2) & $1.850(\mathrm{M} 8.1)$ & M7.5 & M7.5 & $13.9 \pm 1.7$ & 50 \\
\hline 71 & SSSPM J2335-6913 & 1.300 & 6.1 & 0.257 (M7.7) & 2.173 (M6.6) & 1.563 (M6.7) & M7.0 & M7.0 & $42.7 \pm 5.1$ & 37 \\
\hline 72 & APMPM J2344-2906 & 0.975 & 3.0 & 0.121 (M6.9) & 2.161 (M6.4) & $1.530(\mathrm{M} 6.5)$ & M6.0 & M6.5 & $34.1 \pm 4.1$ & 64 \\
\hline 73 & SSSPM J2345-6810 & 1.034 & 0.0 & $0.240(\mathrm{M} 7.6)$ & 2.204 (M6.9) & 1.495 (M6.3) & M7.0 & M7.0 & $43.9 \pm 5.3$ & 46 \\
\hline 74 & APMPM J2347-3154 & 1.094 & 5.6 & 0.263 (M7.7) & $2.322(\mathrm{M} 8.1)$ & 1.924 (M8.4) & M8.5 & M8.0 & $27.2 \pm 3.3$ & 76 \\
\hline 75 & SSSPM J2352-2538 & 1.415 & 6.0 & 0.422 (M8.6) & 2.174 (M6.6) & 2.221 (M9.4) & M9.0 & M9.0 & $16.0 \pm 1.9$ & 31 \\
\hline 76 & SSSPM J2353-4123 & 1.232 & 2.1 & $0.260(\mathrm{M} 7.7)$ & 2.204 (M6.9) & 1.391 (M5.7) & M6.0 & M6.0 & $65.9 \pm 7.9$ & 41 \\
\hline 77 & APMPM J2354-3316 & 2.163 & Flare & 0.774 (L0.6) & 2.158 (Flare) & 1.475 (M6.2) & $\mathrm{M} 8.5^{a}$ & M8.5 & $22.6 \pm 2.7$ & 55 \\
\hline 78 & SSSPM J2356-3426 & 1.310 & 0.0 & 0.557 (M9.4) & $2.359(\mathrm{M} 8.5)$ & 2.076 (M8.9) & M9.0 & M9.0 & $20.0 \pm 2.4$ & 29 \\
\hline 79 & SSSPM J2400-2008 & 1.048 & 0.0 & 0.612 (M9.7) & 2.476 (M9.7) & 2.308 (M9.6) & M9.5 & M9.5 & $35.7 \pm 4.3$ & 110 \\
\hline
\end{tabular}

Note: There are two independent data sets for LP 645-52 and Kelu 1, which are in good agreement. They lead to the same spectral types and corresponding estimates of the distances and tangential velocities.

${ }^{a}$ The spectral type of this object was derived from the quiet spectrum (see Scholz et al. 2004a, for more details).

\section{Subdwarfs found in the survey}

We have identified five subdwarfs among our sample of red high proper motion objects. The spectral classification scheme for these objects, with lower metallicities than those of normal dwarfs, was developed by Reid et al. (1995) and later built upon by Gizis (1997). The latter paper describes the three steps involving $\mathrm{TiO}$ and $\mathrm{CaH}$ band strengths which are used to pin down the spectral type. All objects shown in Fig. 11 fulfil the cutoff criteria defined by Eqs. (4)-(6) in Gizis (1997) which defines them as subdwarfs and from the indices, three objects (LP 614-35, CE 352, SSSPM J0500-5406) are unambiguous extreme subdwarfs with spectral types esdM0.5, esdM3.0, and esdM6.0, respectively.

SSSPM J0500-5406 is among the latest extreme subdwarfs known: the current record holder is the very cool extreme subdwarf, APMPM J0559-2903, at esdM7.0 (Schweitzer et al. 1999). Very cool normal M-type subdwarfs (sdM) have been discovered by Lépine et al. (2003a) (LSR 1425+7102; sdM 8.0) and by Scholz et al. (2004b) (SSSPM J1013-1356; sdM 9.5), and the first $\mathrm{L}$ subdwarfs have recently been published by Burgasser et al. (2003a), Lépine et al. (2003b), Burgasser et al. (2004), and Scholz et al. (2004c).

One of the objects found here, LP 815-21, lies in the boundary region between subdwarfs and extreme subdwarfs. A direct comparison with the spectrum of LHS 489 (esdM0.0; Gizis 1997) establishes their similarity and allows us to classify LP815-21 also as an esdM0.0 object. Another problematic object is LP 314-67, where one of the three indices defined by Gizis (1997) locates it between sdM and esdM, one defines it as sdM, and the last as esdM: again, direct comparison with a similar spectrum was necessary to yield a final spectral type of sdM 3.5.

We have compared the measured colours for each subdwarf with the colours predicted by the evolutionary models for low-metallicity dwarfs of Baraffe et al. (1997) in order to make a preliminary estimate of the metallicity and mass for each source, assuming a typical age of 10 Gyr (Fig. 12). The derived metallicities tend to be higher than the assumed metallicity of -1.2 and -2.0 for subdwarfs and extreme subdwarfs proposed by Gizis (1997). Assuming the metallicities from Fig. 12, we have estimated masses of 0.09-0.10, 0.11, $0.15,0.10$, and $0.20 M_{\odot}$ for SSSPM J0500-5406, LP 314-67, LP 614-35, CE 352, and LP 815-21, respectively. By comparing the observed magnitudes, metallicities, and masses to the magnitudes predicted by evolutionary tracks, we have derived distances of $63 \pm 9,143 \pm 23,264 \pm 15,130 \pm 23$, and $350 \pm 49 \mathrm{pc}$ for SSSPM J0500-5406, LP 314-67, LP 614-35, CE 352, and LP 815-21, respectively, assuming an error of $15 \%$ for these model-dependent distances.

As a check, we have also used comparison objects with known spectral types and trigonometric parallaxes drawn from Gizis (1997). By comparing the 2MASS apparent $J H K_{\mathrm{s}}$ magnitudes for our new subdwarfs with those for a Gizis (1997) 
Table 6. Near-infrared indices and their associated spectral types derived for the objects observed spectroscopically either with VLT/ISAAC or NTT/SOFI. The names of the indices refer to the following papers: Reid et al. (2001) (hereafter R01); Tokunaga \& Kobayashi (1999) (hereafter TK99); and Martín (2000) (hereafter M00). The mean near-infrared spectral type corresponds to the mean value of the four indices. As discussed in the text and as seen in Fig. 10, the discrepancy between near-infrared and optical classifications is large. Thus where an optically-derived spectral type exists for a given source, we have adopted it. When not (sources marked with note ${ }^{a}$ ), we have used the spectral type determined by direct comparison of the near-infrared spectrum with template spectra and/or objects observed with the same instrument setup, where the spectral types of the latter were derived from the optical classification schemes. Preliminary distance estimates based on the near-infrared spectral types are only given for those objects for which a more accurate optical spectral type is not yet available.

\begin{tabular}{|c|c|c|c|c|c|c|c|c|c|}
\hline \multirow[t]{2}{*}{$\mathrm{RN}$} & \multirow[t]{2}{*}{ Name } & \multicolumn{2}{|c|}{ R01 } & \multirow{2}{*}{$\begin{array}{c}\text { TK99 } \\
\text { K1 }\end{array}$} & \multirow{2}{*}{$\begin{array}{c}\text { M00 } \\
Q_{H \text { band }}\end{array}$} & \multirow{2}{*}{$\begin{array}{c}\text { Mean } \\
\text { NIR SpT }\end{array}$} & \multirow{2}{*}{$\begin{array}{c}\text { Adopted } \\
\text { SpT (optical) }\end{array}$} & \multirow{2}{*}{\begin{tabular}{|c|} 
Distance \\
from NIR SpT \\
{$[\mathrm{pc}]$}
\end{tabular}} & \multirow{2}{*}{$\begin{array}{c}v_{\mathrm{t}} \\
{\left[\mathrm{km} \mathrm{s}^{-1}\right]}\end{array}$} \\
\hline & & $\mathrm{H}_{2} \mathrm{O}^{\mathrm{A}}$ & $\mathrm{H}_{2} \mathrm{O}^{\mathrm{B}}$ & & & & & & \\
\hline 04 & SSSPM J0030-3427 & 0.80 (M7.6) & 0.86 (M9.4) & -0.06 (M5.9) & 0.55 (M9.0) & M8.0 & M9.0 $0^{a}$ & $30.4 \pm 3.6$ & 43 \\
\hline 07 & SSSPM J0109-5101 & 0.66 (L2.1) & $0.77(\mathrm{~L} 1.5)$ & 0.22 (L2.1) & $0.42(\mathrm{~L} 1.1)$ & $\mathrm{L} 2.0$ & M8.5 & & \\
\hline 08 & SSSPM J0109-4955 & 0.75 (M9.3) & 0.84 (M9.9) & 0.25 (L2.7) & $0.40(\mathrm{~L} 1.5)$ & $\mathrm{L} 1.0$ & $\mathrm{M} 8.0^{a}$ & $30.8 \pm 3.7$ & 23 \\
\hline 10 & SSSPM J0124-4240 & 0.78 (M8.3) & $0.83(\mathrm{~L} 0.0)$ & 0.10 (M9.3) & 0.52 (M9.5) & L2.5 & ${\mathrm{L} 0.5^{a}}^{a}$ & $17.3 \pm 2.1$ & 22 \\
\hline 11 & SSSPM J0125-6546 & 0.76 (M8.9) & $0.94(\mathrm{M} 7.2)$ & $0.02(\mathrm{M} 7.6)$ & $0.63(\mathrm{M} 7.6)$ & M8.0 & $\mathrm{M} 7.0^{a}$ & $54.0 \pm 6.5$ & 33 \\
\hline 12 & SSSPM J0134-6315 & $0.80(\mathrm{M} 7.8)$ & $0.78(\mathrm{~L} 1.2)$ & 0.22 (L2.0) & 0.49 (M9.9) & L0.0 & $\mathrm{M} 8.0^{a}$ & $47.9 \pm 5.7$ & 26 \\
\hline 14 & SSSPM J0204-3633 & 0.77 (M8.7) & 1.00 (M5.9) & 0.03 (M7.8) & $0.61(\mathrm{M} 8.0)$ & M7.5 & M7.5 & & \\
\hline 16 & APMPM J0207-3722 & $0.80(\mathrm{M} 7.8)$ & $0.83(\mathrm{~L} 0.0)$ & $0.02(\mathrm{M} 7.7)$ & 0.52 (M9.5) & M8.5 & M7.0 & & \\
\hline 17 & SSSPM J0215-4804 & 0.81 (M7.4) & $0.90(\mathrm{M} 8.2)$ & 0.02 (M7.6) & 0.50 (M9.9) & M8.5 & $\mathrm{M} 8.0^{a}$ & $30.9 \pm 3.7$ & 52 \\
\hline 18 & SSSPM J0219-1939 & 0.60 (M5.7) & 0.69 (L3.4) & 0.16 (L0.7) & 0.41 (L1.3) & $\mathrm{L} 2.5$ & L1.0 & & \\
\hline 23 & SSSPM J0306-3648 & 0.73 (L0.0) & 0.87 (M9.1) & 0.09 (M9.1) & 0.44 (L0.8) & L0.0 & M8.0 & & \\
\hline 26 & LP $888-18$ & 0.74 (M9.7) & 0.86 (M9.3) & 0.08 (M9.0) & 0.45 (L0.6) & M9.5 & M8.0 & & \\
\hline 27 & LP 944-20 & 0.63 (L3.2) & 0.79 (L0.9) & 0.11 (M9.6) & 0.38 (L1.9) & L1.5 & M9.5 & & \\
\hline 28 & LP 775-31 & 0.71 (L0.6) & $0.82(\mathrm{~L} 0.3)$ & -0.02 (M6.8) & 0.44 (L0.7) & M9.5 & M7.5 & & \\
\hline 29 & LP $655-48$ & 0.77 (M8.6) & 0.89 (M8.6) & 0.06 (M8.5) & 0.53 (M9.2) & M8.5 & M7.5 & & \\
\hline 42 & Kelu 1 & 0.66 (L2.2) & 0.75 (L2.1) & 0.28 (L3.2) & 0.35 (L2.3) & L2.5 & L2.0 & & \\
\hline 56 & [HB88] M12 & 0.85 (M6.2) & 1.00 (M5.8) & 0.00 (M7.2) & 0.65 (M7.2) & M6.5 & M4.5 & & \\
\hline 59 & SSSPM J2229-6931 & 0.80 (M7.6) & 0.88 (M8.7) & $-0.14(\mathrm{M} 4.2)$ & $0.55(\mathrm{M} 8.9)$ & M7.5 & $\mathrm{L} 0.0^{a}$ & $34.4 \pm 4.1$ & 36 \\
\hline 65 & SSSPM J2307-5009 & 0.65 (L2.5) & $0.78(\mathrm{~L} 1.2)$ & 0.03 (M7.8) & 0.40 (L1.4) & L0.5 & M9.0 & & \\
\hline 66 & SSSPM J2310-1759 & $0.72(\mathrm{~L} 0.3)$ & $0.78(\mathrm{~L} 1.4)$ & 0.13 (L0.0) & 0.37 (L2.0) & $\mathrm{L} 1.0$ & M9.5 & & \\
\hline 67 & SSSPM J2319-4919 & 0.75 (M9.2) & 0.96 (M6.7) & -0.03 (M6.6) & $0.62(\mathrm{M} 7.9)$ & M7.5 & $\mathrm{M}_{8} .0^{a}$ & $33.9 \pm 4.1$ & 35 \\
\hline 69 & APMPM J2330-4737 & 0.75 (M9.5) & 0.97 (M6.6) & 0.08 (M8.9) & 0.54 (M9.1) & M8.5 & M6.0 & & \\
\hline 70 & APMPM J2331-2750 & 0.72 (L0.4) & 0.93 (M7.6) & 0.13 (L0.0) & 0.53 (M9.3) & M9.5 & M7.5 & & \\
\hline 73 & SSSPM J2345-6810 & 0.71 (L0.7) & $1.04(\mathrm{M} 4.8)$ & $0.06(\mathrm{M} 8.5)$ & $0.64(\mathrm{M} 7.4)$ & M8.0 & M7.0 & & \\
\hline 75 & SSSPM J2352-2538 & $0.70(\mathrm{~L} 1.0)$ & $0.92(\mathrm{M} 7.9)$ & 0.10 (M9.3) & 0.48 (L0.1) & M9.5 & M9.0 & & \\
\hline 78 & SSSPM J2356-3426 & $0.62(\mathrm{~L} 3.5)$ & $0.81(\mathrm{~L} 0.5)$ & $-0.10(\mathrm{M} 5.1)$ & $0.30(\mathrm{~L} 3.1)$ & L0.5 & M9.0 & & \\
\hline 79 & SSSPM J2400-2008 & 0.63 (L3.1) & $0.82(\mathrm{~L} 0.4)$ & 0.07 (M8.7) & 0.34 (L2.5) & $\mathrm{L} 1.0$ & M9.5 & & \\
\hline
\end{tabular}

source with a matching spectral type, we are again able to estimate the distances for our sources. We have also used the empirical relationships between spectral types and absolute $M_{K_{\mathrm{s}}}$ magnitudes for $\mathrm{sdM}$ and esdM objects given by Lépine et al. (2003c). For the four new extreme subdwarfs, the bootstrapped distance estimates agree with the model-dependent estimates to within the errors, while the bootstrapping technique yields significant differences for the subdwarf LP 314-67, as seen in Table 7.

Armed with these distance estimates and the proper motions for each source, we only require the radial velocity before we are able to calculate the true space velocities. We have been able to use the relatively sharp Ca II lines at 8542 and $8662 \AA$ to detect radial velocity shifts in the five subdwarfs. The measured shifts were $+3.5,+8.0,-2.5,+6.5$, and $+7.0 \pm 0.5 \AA$, corresponding to heliocentric radial velocities of $+247,+214,+288$, -66 , and $+122 \pm 20 \mathrm{~km} \mathrm{~s}^{-1}$ for SSSPM J0500-5406, LP 314-67, LP 614-35, CE 352, and LP 815-21, respectively.

Finally then, we are able to compute the heliocentric space velocities following Johnson \& Soderblom (1987), doing so for each distance estimation technique as described above. As listed in Table 7, the determined space velocity components are 
Table 7. Spectral indices and associated spectral types (with an accuracy of half a subclass), distance estimates, and kinematics for the five subdwarfs discovered in our survey.

\begin{tabular}{|c|c|c|c|c|c|c|c|c|c|c|c|c|}
\hline $\mathrm{RN}$ & Name & TiO5 & $\mathrm{CaH} 1$ & $\mathrm{CaH} 2$ & $\mathrm{CaH} 3$ & SpT & $\begin{array}{l}d_{\text {spec }} \\
{[\mathrm{pc}]}\end{array}$ & $\begin{array}{c}v_{\mathrm{t}} \\
{\left[\mathrm{km} \mathrm{s}^{-1}\right]}\end{array}$ & $\begin{array}{c}v_{r} \\
{\left[\mathrm{~km} \mathrm{~s}^{-1}\right]}\end{array}$ & $\begin{array}{c}U \\
{\left[\mathrm{~km} \mathrm{~s}^{-1}\right]}\end{array}$ & $\begin{array}{c}V \\
{\left[\mathrm{~km} \mathrm{~s}^{-1}\right]}\end{array}$ & $\begin{array}{c}W \\
{\left[\mathrm{~km} \mathrm{~s}^{-1}\right]}\end{array}$ \\
\hline \multirow[t]{3}{*}{30} & SSSPM J0500-5406 & 0.984 & 0.355 & 0.270 & 0.382 & esdM6.0 & $63 \pm 09^{a}$ & 313 & $+247 \pm 20$ & $+262 \pm 48$ & $-287 \pm 22$ & $-84 \pm 17$ \\
\hline & & & & & & & $70 \pm 11^{b}$ & 348 & & $+294 \pm 60$ & $-298 \pm 25$ & $-77 \pm 19$ \\
\hline & & & & & & & $54 \pm 08^{c}$ & 268 & & $+220 \pm 43$ & $-274 \pm 21$ & $-94 \pm 16$ \\
\hline \multirow[t]{3}{*}{35} & LP $314-67$ & 0.773 & 0.596 & 0.433 & 0.643 & $\operatorname{sdM} 3.5$ & $143 \pm 23^{a}$ & 314 & $+214 \pm 20$ & $-128 \pm 14$ & $-351 \pm 52$ & $+63 \pm 24$ \\
\hline & & & & & & & $395 \pm 59^{b}$ & 866 & & $-130 \pm 22$ & $-872 \pm 136$ & $-115 \pm 51$ \\
\hline & & & & & & & $193 \pm 29^{c}$ & 423 & & $-128 \pm 15$ & $-454 \pm 71$ & $+27 \pm 30$ \\
\hline \multirow[t]{3}{*}{37} & LP 614-35 & 1.057 & 0.744 & 0.660 & 0.806 & esdM0.5 & $264 \pm 15^{a}$ & 237 & $+288 \pm 20$ & $-168 \pm 35$ & $-261 \pm 24$ & $+206 \pm 19$ \\
\hline & & & & & & & $265 \pm 40^{b}$ & 237 & & $-169 \pm 37$ & $-261 \pm 25$ & $+206 \pm 19$ \\
\hline & & & & & & & $257 \pm 39^{c}$ & 230 & & $-163 \pm 34$ & $-258 \pm 23$ & $+207 \pm 19$ \\
\hline \multirow[t]{3}{*}{44} & CE 352 & 0.871 & - & 0.471 & 0.626 & esdM3.0 & $130 \pm 23^{a}$ & 217 & $-66 \pm 20$ & $-186 \pm 29$ & $-121 \pm 32$ & $-46 \pm 11$ \\
\hline & & & & & & & $195 \pm 29^{b}$ & 325 & & $-258 \pm 65$ & $-200 \pm 71$ & $-52 \pm 12$ \\
\hline & & & & & & & $149 \pm 22^{c}$ & 249 & & $-207 \pm 32$ & $-144 \pm 34$ & $-48 \pm 11$ \\
\hline \multirow[t]{3}{*}{49} & LP $815-21$ & 0.952 & - & 0.773 & 0.870 & esdM0.0 & $350 \pm 49^{a}$ & 340 & $+122 \pm 20$ & $+269 \pm 35$ & $-240 \pm 53$ & $-13 \pm 15$ \\
\hline & & & & & & & $347 \pm 52^{b}$ & 337 & & $+267 \pm 34$ & $-237 \pm 51$ & $-14 \pm 15$ \\
\hline & & & & & & & $278 \pm 42^{c}$ & 270 & & $+233 \pm 30$ & $-181 \pm 42$ & $-23 \pm 13$ \\
\hline
\end{tabular}

Notes: ${ }^{a}$ Model-dependent distance estimate according to Baraffe et al. (1997); ${ }^{b}$ distance estimated using comparison objects from Gizis (1997); ${ }^{c}$ distance based on empirical relationship between spectral types and $M_{K_{\mathrm{S}}}$ in Lépine et al. (2003c).

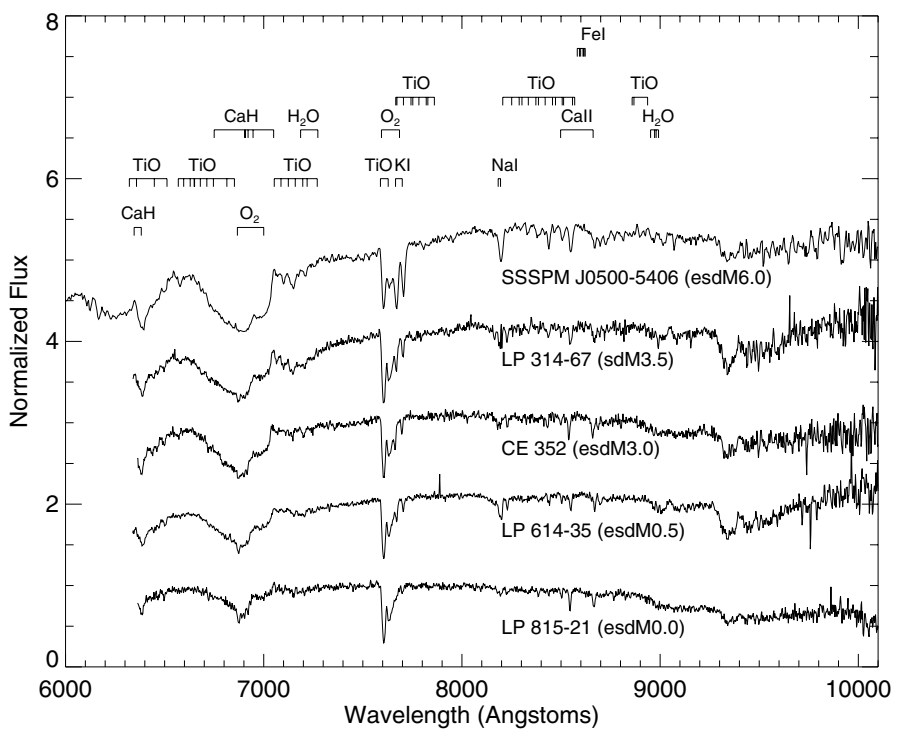

Fig. 11. Optical spectra of the five subdwarfs and extreme subdwarfs found among the proper motion objects observed with VLT/FORS1 and ESO $3.6 \mathrm{~m} / \mathrm{EFOSC} 2$. The classification scheme described in Gizis (1997) was used to determine the spectral types. An arbitrary constant has been added to separate the spectra.

typical of (extreme) subdwarfs (cf. Figs. 17 and 18 in Lépine et al. $2003 \mathrm{c}$ with velocities ranging from 100 to $500 \mathrm{~km} \mathrm{~s}^{-1}$ ). The largest calculated space velocity is that for the sdM $3.5 \mathrm{ob}-$ ject LP 314-67, assuming the distance bootstrapped from the Gizis (1997) comparison source: its velocity is comparable to the largest space velocities for subdwarfs presented by Lépine et al. (2003c).

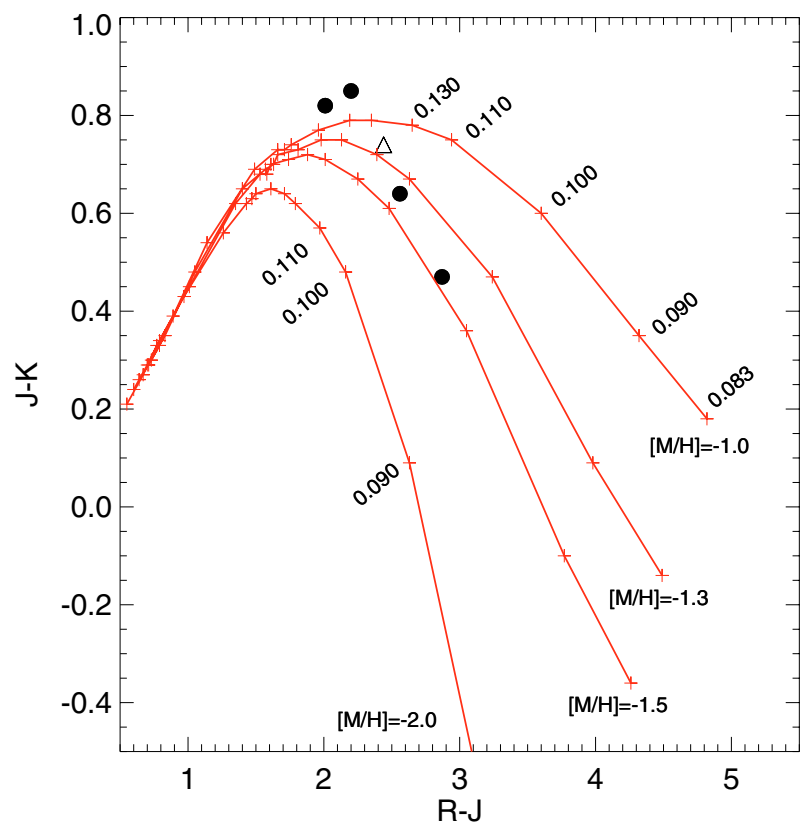

Fig. 12. Colour-colour diagram $(R-J, J-K)$ for the subdwarfs and extreme subdwarfs found in our proper motion sample. The extreme subdwarfs are shown as filled circles while the only subdwarf, LP 314-67, is displayed as a open triangle. The isochrones from Baraffe et al. (1997) for low-metallicity dwarfs ranging from $[\mathrm{Fe} / \mathrm{H}]=-2.0$ to -1.0 are shown for comparison. Crosses from left to right indicate the following masses: $0.80,0.75,0.70,0.60,0.50,0.40,0.35,0.30,0.20$, $0.15,0.13,0.100,0.090,0.085$, and $0.083 M_{\odot}$. The steps for the masses are identical for all metallicities.

\section{Distance estimates for the normal M and $L$ dwarfs}

Our technique for establishing the distances of the normal $\mathrm{M}$ and $\mathrm{L}$ dwarfs in our sample is more straightforward, using the 
absolute $M_{J}$ versus spectral type relationship of Dahn et al. (2002) as follows:

$M_{J}=8.38+0.341 \times \mathrm{SpT}$,

which is valid for spectral types between M6.5 and L8.0, and where the spectral type, $\mathrm{SpT}=6.5$ for M6.5 and increases to 18 for L8. The error in the relation is $\sim 0.25 \mathrm{mag}$ and in computing the distances for the sources in our sample, we have combined that error with a $0.1 \mathrm{mag}$ error in the measured apparent $J$ magnitude. Note that we have used the $J$ magnitudes from 2MASS and not our own ISAAC $J_{\mathrm{s}}$ magnitudes to compute distances.

For objects with spectral types earlier than M6.5, we have employed the absolute magnitudes given by Kirkpatrick \& McCarthy (1994) for all early- and mid-M dwarfs, except for M5.5 dwarfs. For these, we used absolute magnitudes of $M_{J}=9.87, M_{H}=9.31$, and $M_{K \mathrm{~s}}=8.97$ as kindly provided by Hartmut Jahreiß (personal communication), based on the following standards: LHS 2, LHS 39, LHS 549, LHS 1565 , and LHS 3339. For the early type sources in our sample, the distances were determined as an average of those calculated for each of the three near-infrared filters, and the uncertainties were estimated using the assumptions described above for the late-type objects.

The resulting distance estimates are listed in Tables 5 and 6. The great majority of the late-type (>M6) dwarfs appear to be located within $50 \mathrm{pc}$ of the Sun, with 24 falling in the range of the catalogue of nearby stars $(<25 \mathrm{pc})$ and 11 of those within $15 \mathrm{pc}$. On the other hand, the majority of the earlier-type objects are located at larger distances ranging from 100-200 pc: only three of them are members of the $25 \mathrm{pc}$ sample.

\section{Kinematics and activity}

Combining the distance estimates with the measured proper motions, the tangential velocities of the sources can be calculated (Tables 5 and 6). The large distances estimated for most of the early-type M dwarfs (<M5.5) and the relatively large proper motions measured in some cases lead to extremely large tangential velocities of $\sim 200-300 \mathrm{~km} \mathrm{~s}^{-1}$ (Fig. 13), comparable with those of the subdwarfs described earlier (Table 7). The most extreme example is the M4.5 dwarf, APMPM J2036-4936, with a tangential velocity of $335 \mathrm{~km} \mathrm{~s}^{-1}$. Such large velocities are more typical of metal-poor stars than of metal-rich ones, although Lépine et al. (2003c) have also reported several normal $\mathrm{M}$ dwarfs which may be members of the Galactic halo population. Gizis (1997) described a number of sources with early M-type spectra and halo kinematics which he classified as normal $\mathrm{M}$ dwarfs, rather than sdM or esdM subdwarfs. Indeed such objects are to be expected, since his classification scheme sources with metallicities of $\approx-0.5$ are normal $\mathrm{M}$ dwarfs, not subdwarfs, and the metallicity distribution of halo stars extends down to this level. Among the later-type (>M5.5) dwarfs, the tangential velocities are typically substantially less than $100 \mathrm{~km} \mathrm{~s}^{-1}$, with the M9.5 dwarf, SSSPMJ2400-2008, the most extreme case at $110 \mathrm{~km} \mathrm{~s}^{-1}$.

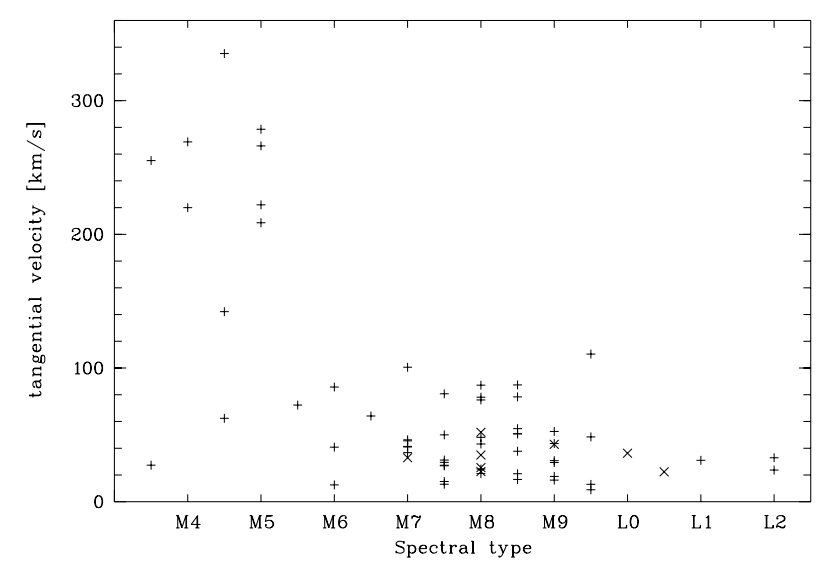

Fig. 13. Tangential velocities vs. spectral types for all spectroscopically classified (+ optical, $\times$ near-infrared) $\mathrm{M}$ and $\mathrm{L}$ dwarfs in this study.

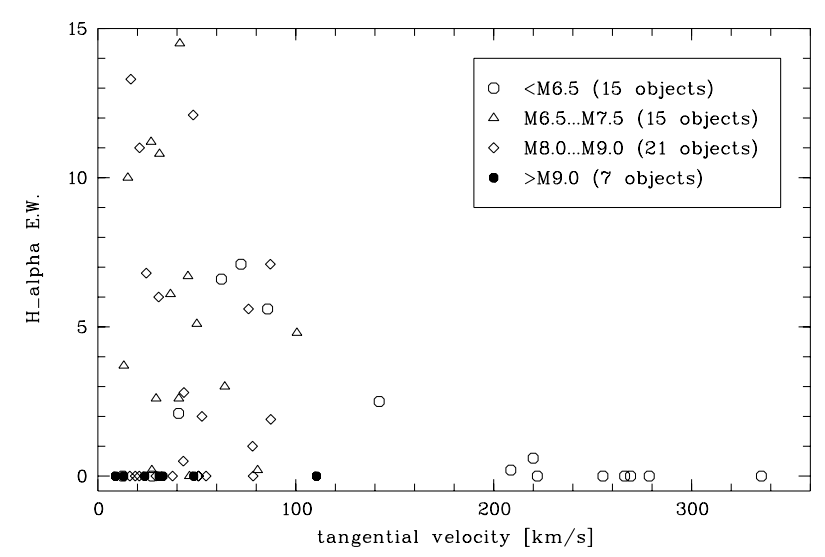

Fig. 14. H $\alpha$ equivalent widths vs. tangential velocities for all $\mathrm{M}$ dwarfs in the present study.

Gizis et al. (2000) have demonstrated a relationship between tangential velocity and chromospheric activity for late (M8-M9.5) dwarfs, inasmuch as all objects with $\mathrm{H} \alpha$ equivalent widths over $10 \AA$ have tangential velocities greater than $20 \mathrm{~km} \mathrm{~s}^{-1}$ and up to $80 \mathrm{~km} \mathrm{~s}^{-1}$. The velocity dispersion of a population of stars increases with age as they interact with the potential of the galactic disk and thus low velocity stars are generally younger than high velocity stars. The usual prejudice is that young, more rapidly rotating stars exhibit more chromospheric activity than their older, spun-down counterparts, but the Gizis et al. (2000) finding shows that in fact the opposite applies to ultracool dwarfs, where the activity declines due to the very low temperatures.

There appears to be a second order effect however, namely that activity is also correlated with mass. For a given ultracool spectral type (e.g., late $\mathrm{M}$ or L), a sample of sources may contain both old and young objects: the old objects will be true stellar sources (i.e., above $\sim 0.075 M_{\odot}$ ) which have cooled and contracted down to their main sequence parameters, while the younger sources could well be substellar objects (i.e., below $\sim 0.075 M_{\odot}$ ) which are still deriving luminosity and an enhanced effective temperature from their pre-main sequence contraction. These latter sources will be slightly larger and more luminous, and have a lower surface gravity than their 
older, same-spectral-type counterparts. Gizis et al. (2000) suggest that this may lead to the observed reduction in chromospheric activity.

With our new sample of ultracool dwarfs, we can explore these relationships a little further, although it must be emphasised that we cannot do so in a statistical sense. Our sample is proper-motion selected and is therefore biased towards older, high velocity objects and away from younger, lower velocity sources.

We have used two different indices to estimate the level of chromospheric activity for the objects in our sample. First, we have computed the $\mathrm{H} \alpha$ index defined by Kirkpatrick et al. (1999; see their Table 4), which measures the strength of the $\mathrm{H} \alpha$ emission line compared to the neighbouring continuum: values above 1 indicate significant emission. Second, we have measured the equivalent width of the $\mathrm{H} \alpha$ line. The results of both measurements are given in Table 5 .

First, we note that there is a peak in activity around spectral types M6-M7, as also reported by Hawley et al. (1996) and Gizis et al. (2000) for larger samples of nearby M dwarfs. In more detail, we see that there are seven very active objects with $\mathrm{H} \alpha$ equivalent widths larger than $10 \AA$, all of which are M7-M8.5 dwarfs. Interestingly, these sources all have relatively small tangential velocities, $v_{\mathrm{t}}<50 \mathrm{~km} \mathrm{~s}^{-1}$, three of them below $20 \mathrm{~km} \mathrm{~s}^{-1}$ : their average velocity is roughly half that of the full population of M7-M8.5 sources in our sample.

Thus, at first sight, this subsample appears to contradict the finding of Gizis et al. (2000), namely that active, very late type sources tended to have larger velocities than their inactive counterparts. However, when we more rigorously compare like with like, we find that their conclusion remains valid. If we consider only the M8-M9.5 sources and divide them into $\mathrm{H} \alpha$ active (EW $\geq 0.5 \AA$; 12 sources) and inactive ( $\mathrm{EW}<0.5 \AA$; 13 sources) samples, we find mean tangential velocities of 52 and $40.5 \mathrm{~km} \mathrm{~s}^{-1}$, respectively. That is, the active sources have higher velocities on average, consistent with the finding of Gizis et al. (2000).

\section{Discussion related to specific objects}

\subsection{L dwarfs}

In Lodieu et al. (2002), we described the discovery of three new early L dwarfs from our proper motion survey, with optical and near-infrared spectroscopy for two of them (SSSPM J2310-1759, SSSPM J0219-1939) and near-infrared spectroscopy alone for the third (SSSPM J0109-5101). The spectral types derived by Lodieu et al. (2002) based on nearinfrared spectral indices were L1, L2, and L2, respectively, but we now find that these results must be revised.

We have made direct comparisons of the optical spectra which we now have for all three sources with template stars observed with the same instrumental set-up, deriving revised spectral types of M9.5, L1.0, and M8.5 for SSSPM J2310-1759, SSSPM J0219-1939, and SSSPM J0109-5101, respectively, with uncertainties of half a subclass. These differ significantly from the previous results, in line with the trend shown in Fig. 10, where the near-infrared spectral classification tends to yield a later spectral types than found from the optical spectra.

As a consequence of the revised spectral types, the corresponding spectroscopic parallaxes shown in Table 5 have also been changed, although the objects remain relatively nearby: SSSPM J2310-1759 and SSSPM J0219-1939 lie within 30 pc, while SSSPM J0109-5101 may be within 15 pc.

The nearest L dwarf in our sample (SSSPM J0829-1309; L2.0) has a spectroscopic distance of $\sim 12 \mathrm{pc}$ and we confirm the spectral type obtained from a lower signal-to-noise spectrum in the discovery paper by Scholz \& Meusinger (2002). Its optical spectrum is shown in Fig. 5 alongside the very similar spectrum for Kelu 1.

Finally, there are two other L dwarf candidates (SSSPM J0124-4240 at L0.5; SSSPM J2229-6931 at L0.0) in our sample for which, however, only near-infrared spectra could be used for classification (see Fig. 6). Given the apparent systematic shift in spectral types between optical and near-infrared spectra for the sources discussed above and as similarly seen for Kelu 1, LP 944-20, and SSSPM J2400-2008, these early L dwarf classifications for SSSPM J0124-4240 and SSSPM J2229-6931 must be regarded with some caution: it is entirely possible that they will be reclassified as late $\mathrm{M}$ dwarfs once appropriate optical spectra are obtained.

\subsection{Nearby $M$ dwarfs}

Our sample contains two late $M$ dwarfs within $10 \mathrm{pc}$, LP775-31 and LP 655-48, which we originally classifed as M8.0 and M7.5, respectively, on their discovery (McCaughrean et al. 2002). Cruz \& Reid (2002) had earlier claimed that both sources were M6 dwarfs, but Cruz et al. (2003) subsequently reclassified both as $\mathrm{M} 7$. We have also revisited their optical spectra as part of the present work and adopt spectral types of M7.5 for both (Table 5), consistent with the Cruz et al. (2003) results to within the errors. In either case, the distances nominally stay below $10 \mathrm{pc}$, but as pointed out by Cruz et al. (2003), trigonometric parallaxes are needed to settle the matter.

However, it is worth noting that the near-infrared spectra for these two sources differ significantly, with LP 655-48 being redder in the range $1.46-1.69 \mu \mathrm{m}$, redder from $2.0-2.2 \mu \mathrm{m}$, bluer from 2.2-2.4 $\mu \mathrm{m}$, and exhibiting a stronger $\mathrm{CO}$ bandhead break. As recently demonstrated by Close et al. (2003), these differences may indicate that one or both of the objects may be a binary with a very late type companion.

In addition to these very nearby sources, there are several other $\mathrm{M}$ dwarfs in our sample which we find to be rather nearby, including LP 888-18 and APMPM J1251-2121 at 11 pc, and SSSPM J0829-1309 at 12 pc.

\subsection{Objects with proper motions larger than 1 arcsec per year}

Four of the discoveries presented in this study have proper motions exceeding $1 \operatorname{arcsec} \mathrm{yr}^{-1}$. One of these objects, SSSPM J0500-5406, was spectroscopically classified as an ultracool subdwarf (esdM6.0), while the other 
three are mid-M dwarfs: SSSPM J1926-4311 (M5.0), APMPM J1957-4216 (M5.0), and APMPM J2330-4737 (M6.0). The large tangential velocities $\left(>200 \mathrm{~km} \mathrm{~s}^{-1}\right)$ implied for SSSPM J1926-4311 and APMPM J1957-4216 suggest that they may well be members of the Galactic halo population, while the derived $v_{\mathrm{t}} \sim 90 \mathrm{~km} \mathrm{~s}^{-1}$ for APMPM J2330-4737 may make it a representative of the thick disk. The identification of a relatively large number of these relatively rare objects in this study simply confirms the strong bias towards them that naturally results from proper motion based searches (see also Scholz et al. 2004b).

\subsection{Objects with blue colours}

There are two objects in our sample with unusually blue colours for their spectral types, even after considering the rather large dispersion in the colours originating with the relatively poor accuracy of the photographic SSS magnitudes (see Fig. 2). The $B_{J}-R$ colour of the first of these objects, SSSPM J0027-5402, is $\sim 1.1$, whereas its M7 spectral type would predict something closer to 2.5 . None of the other colours for this source seem out of character with its spectral type which would, at first sight, suggest a photometric error in $B_{J}$, despite its apparent brightness at that wavelength. An alternative solution might be the presence of a very blue companion or chance aligned field star.

The second source, SSSPM J0134-6315, has been classified as M8, but its $I-J \sim 1.5$ and $R-K_{\mathrm{S}} \sim 5.3$ are more consistent with something closer to M5. In this case, the explanation may lie with the fact that we have only a near-infrared spectrum for this source, which may again have skewed our classification towards a later type than would be derived in the optical, as discussed above.

There are two other objects labelled in Fig. 2 with nominally erroneous colours: these are not members of our sample, but are shown for comparison. One is 2MASS 1411-21, classified as an M9 dwarf by both Cruz et al. (2003) and Kendall et al. (2004), but also appearing very blue in the optical with $B_{J}-R \sim 2.0$. Again, a blue companion or aligned field star might be the explanation. The other source, SDSS 1326-00 (Fan et al. 2000) has an uncertain spectral type of "L8?" but was barely detected in the SSS I band data and has a very small $I-J$ colour of 2.2, much below the expected $\sim 3.5$. The proper motion for this source of $(-294,-1027) \pm(20,3)$ mas yr $^{-1}$ obtained from one SSS, one 2MASS, and one SDSS position formally supports its identification, but there remains room for error, as the epochs of the three observations were not well distributed (SSS 1991.256; 2MASS 1999.093; SDSS 1999.219). This object requires further investigation and at the very least, a new epoch position would prove very helpful in verifying the proper motion and the corresponding SSS identification.

\subsection{Common proper motion stars}

One of the new high proper motion objects was found to be a common proper motion companion 32 arcsec from the previously known LHS 3141 and has therefore been named
LHS 3141B. The catalogued proper motion of the primary, $(+204,-519) \pm(5,8)$ mas $\mathrm{yr}^{-1}$, agrees to within the errors with our measured proper motion for LHS 3141B. We have classified this latter object as an M7 dwarf, but unfortunately, no spectral type presently exists for the brighter primary. According to its optical-to-infrared colours $(I-J \sim 1.1$; $R-K_{\mathrm{s}} \sim 3.4$ ), it should be an early-type $\mathrm{M}$ dwarf. Assuming a distance of $37 \mathrm{pc}$ as derived from the spectroscopic parallax for the secondary, the projected physical separation is $\sim 1200 \mathrm{AU}$.

The previously known common proper motion pair LDS 4980 was included in our spectroscopic program due to the relatively red colour measured from APM scans of the POSS- $1 O$ and $E$ plates. The spectral types determined for the pair, M3.5 and M4, are rather early and imply a relatively large distance of $\sim 210 \mathrm{pc}$, in good agreement with independent estimates for the two components. The measured angular separation of about 79 arcsec between the pair then translates into a large projected physical separation of $\sim 16600 \mathrm{AU}$ or $0.08 \mathrm{pc}$. The proper motions of the two components also agree within the errors and the implied tangential velocity appears to be in excess of $200 \mathrm{~km} \mathrm{~s}^{-1}$, rather large for M dwarfs and indicating membership in the Galactic halo.

Finally, our sample contains another new common proper motion component to a known system, APMPM J2354-3316, recently been described in detail by Scholz et al. (2004a). It is a wide M8.5 companion to the M4/DA binary LHS 4039/LHS 4040 and was found to show very strong $\mathrm{H} \alpha$ emission line and a blue continuum below $7500 \AA$.

\subsection{Objects without spectroscopy}

As discussed in Sect. 3, eight of the objects in our sample have not yet been observed spectroscopically at either optical or near-infrared wavelengths, although imaging observations were made for all of them using the VLT.

Five of these objects (APMPM J0207-7214, APMPM J0244-5203， LHS 2555a， APMPM J0331-2349, APMPMJ1212-2126) have small optical-to-infrared indices $\left(R-K_{\mathrm{S}}<4\right.$ and $\left.I-J<1.5\right)$ typical of earlyM dwarfs (cf. Fig. 2) or subdwarfs (Sect. 5). The other three objects (APMPM J0057-7604, APMPM J0232-4437, APMPM J0536-5358) have larger indices $\left(5.2<R-K_{\mathrm{S}}<6.2\right.$ and $I-J \sim 2.1)$ similar to the spectroscopically-classified M5-M7 dwarfs in our sample. The brightest of these objects, APMPM J0057-7604 with $K_{\mathrm{s}} \sim 11.6$, is most likely located within $50 \mathrm{pc}$ and could be within $25 \mathrm{pc}$ sample if its spectral type turns out to be later than M6.

\section{Summary and outlook}

We have presented optical and near-infrared photometry and spectroscopy for a sample of 71 red proper motion objects and we have classified 58 and 27 of them using optical and near-infrared spectroscopy, respectively; the remaining eight sources have optical and near-infrared photometry only.

The spectroscopic sample comprises $60 \mathrm{M}$ dwarfs, $6 \mathrm{~L}$ dwarfs, and 5 subdwarfs. For the sources with both optical and near-infrared spectroscopy, we have observed a systematic 
shift in the near-infrared of about one subtype towards later spectral types. As a consequence, we have favoured the optical spectral types whenever possible for consistency with other approaches in the literature. Partly in connection with this, we have revisited the spectral typing of three previously published $\mathrm{L}$ dwarfs and two M dwarfs in the course of this work, generally finding them to have earlier types than before.

The large majority of the late-type dwarfs (spectral types later than M6) are located within $50 \mathrm{pc}$ of the Sun and generally have small tangential velocities $\left(<50 \mathrm{~km} \mathrm{~s}^{-1}\right)$, whereas the early-type $\mathrm{M}$ dwarfs tend to have larger distances ranging from $100-200 \mathrm{pc}$ and tangential velocities in the range $100-200 \mathrm{~km} \mathrm{~s}^{-1}$. These correlations, of course, simply reflect the search strategy which we have employed, which is fluxand proper motion-limited. Intrinsically fainter sources can be nearby and need only have relatively low velocities; brighter, earlier-type stars can be picked up at larger distances, as long as they have larger space velocities. We see the previously known peak in chromospheric activity around M6-M7 and also provide mild confirmation for the finding by Gizis et al. (2000) of a correlation between tangential velocity and chromospheric activity over the restricted spectral type range M8-M9.5. However, as discussed in Sect. 7, our proper motion selection strategy is biased towards older, higher velocity sources, making it hard to assign any kind of statistical significance to the reality of this effect.

Among the subdwarfs in the sample, four are classified as extreme subdwarfs and one of these, SSSPM 0500-5406 is among the coolest $\mathrm{M}$ subdwarfs discovered to date with an estimated spectral type of esdM6. The fact that we have not discovered any new L subdwarfs perhaps suggests that Population II may be deficient in substellar objects with respect to Population I, i.e., the local field. This is contrary to expectations: very metal-poor gas has a reduced dust cooling efficiency (less effective gas-to-dust coupling) and thus should yield smaller minimum Jeans mass fragments. These should then be able to collapse to form brown dwarfs before they merge into larger objects, and thus in theory yield large numbers of substellar objects (Zinnecker 1995). However, the search strategies for Population II brown dwarfs (substellar subdwarfs) still suffer from various selection biases, with the general problem of identifying very large proper motions at the magnitude limit of optical surveys and difficulties in optimising the colour search parameters.

Finally, we have identified several interesting new sources worthy of more detailed follow-up observations, including trigonometric parallax measurements, spectroscopy in the lithium line to search for evidence of a substellar nature, searches for low-mass companions, and so on. Three of the new objects lie at close to $10 \mathrm{pc}$; four objects have proper mo-

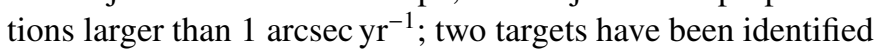
as common proper motion companions to previously known nearby objects; and some objects exhibit unusual blue colours, which might indicate the presence of a companion.

Acknowledgements. Our survey for new, faint, high proper motion objects is based on the SuperCOSMOS Sky Surveys, i.e., digitised data obtained from scans of UKST and ESO Schmidt plates. We would like to thank the SuperCOSMOS team for producing such excellent data. All of the new observations presented in this paper were obtained with ESO's telescopes on Paranal and La Silla, and we acknowledge the expertise of, advice from, and enriching discussions with the ESO support teams during the various runs. We thank the referee, John Gizis, for his kind report and some useful comments. This research has also made use of data products from the Two Micron All Sky Survey, which is a joint project of the University of Massachusetts and the Infrared Processing and Analysis Center, funded by the National Aeronautics and Space Administration and the National Science Foundation, as well as of the VizieR catalogue access tool, CDS, Strasbourg. We thank Gyula Szokoly for obtaining low-resolution observations of the objects APMPM J2036-4936 and LP 859-1 for us. Finally, N.L. and M.J.M. thank the European Research Training Network on "The Formation and Evolution of Young Stellar Clusters" (HPRN-CT-2000-00155) for financial support.

\section{References}

Baraffe, I., Chabrier, G., Allard, F., \& Hauschildt, P. H. 1998, A\&A, 327,1054

Burgasser, A. J., Kirkpatrick, J. D., \& Reid, I. N. 2000, AJ, 120, 473

Burgasser, A. J., Kirkpatrick, J. D., \& Brown, M. E. 2002, ApJ, 564, 421

Burgasser, A. J., Kirkpatrick, J. D., Burrows, A., et al. 2003a, ApJ, 592,1186

Burgasser, A. J., Kirkpatrick, J. D., McElwain, M. W., et al. 2003b, $\mathrm{AJ}, 125,850$

Burgasser, A. J., McElwain, M. W., \& Kirkpatrick, J. D. 2003c, AJ, 126, 2487

Burgasser, A. J., McElwain, M. W., Kirkpatrick, J. D., et al. 2004, AJ, 127,2856

Burgasser, A. J. 2004, ApJ, 614, 73

Close, L. M., Siegler, N., Freed, M., \& Biller, B. 2003, ApJ, 587, 407

Cruz, K. L., \& Reid, I. N. 2002, AJ, 123, 2828

Cruz, K. L., Reid, I. N., Liebert, J., et al. 2003, AJ, 126, 2421

Dahn, C. C., Harris, H. C., Vrba, F. J., et al. 2002, AJ, 124, 1170

Delfosse, X., Forveille, T., Martín, E. L., et al. 2001, A\&A, 366, L13

Digby, A. P., Hambly, N. C., Cooke, J. A., et al. 2003, MNRAS, 344, 583

Epchtein, N., de Batz, B., Capoani, L., et al. 1997, Msngr, 87, 27

Fan, X., Knapp, G. R., Strauss, M. A., et al. 2000, AJ, 119, 928

Geballe, T. R., Knapp, G. R., Leggett, S. K., et al. 2002, ApJ, 564, 466

Gizis, J. E. 1997, AJ, 113, 806

Gizis, J. E., Monet, D. G., Reid, I. N., et al. 2000, AJ, 120, 1085

Gliese, W., \& Jahreiß, H. 1991, Preliminary Version of the Third Catalogue of Nearby Stars, ADC Selected Astronomical Catalogs, Vol. 1, CD-ROM

Goldman, B., Delfosse, X., Forveille, T., et al. 1999, A\&A, 351, L5

Hambly, N. C., MacGillivray, H. T., Read, M. A., et al. 2001a, MNRAS, 326, 1279

Hambly, N. C., Irwin, M. J., \& MacGillivray, H. T. 2001b, MNRAS, 326, 1295

Hambly, N. C., Davenhall, A. C., Irwin, M. J., \& MacGillivray, H. T. 2001c, MNRAS, 326, 1315

Hambly, N. C., Henry, T., Subasavage, J., Brown, M., \& Jao, W.-C. 2004, AJ, 128, 437

Hawkins, M. R. S., \& Bessell, M. S. 1988, MNRAS, 237, 177

Hawley, S. L., Gizis, J. E., \& Reid, I. N. 1996, AJ, 112, 2799

Henry, T. J., Ianna, P. A., Kirkpatrick, J. D., et al. 1997, AJ, 114, 388

Henry, T. J., Walkowicz, L. M., Barto, T. C., \& Golimowski, D. A. 2002, AJ, 123, 2002

Ianna, P. A., \& Fredrick, L. W. 1995, ApJ, 441, L47 
Johnson, D. R. H., \& Soderblom, D. R. 1987, AJ, 93, 864

Kendall, T. R., Delfosse, X., Martín, E. L., \& Forveille, T. 2004, A\&A, 416, L17

Kirkpatrick, J. D., \& McCarthy, D. W. 1994, AJ, 107, 333

Kirkpatrick, J. D., Henry, T. J., \& Irwin, M. J. 1997, AJ, 113, 1421

Kirkpatrick, J. D., Reid, I. N., Liebert, J., et al. 1999, ApJ, 519, 802

Kirkpatrick, J. D., Reid, I. N., Liebert, J., et al. 2000, AJ, 120, 447

Leggett, S. K., Geballe, T. R., \& Fan, X. 2000, ApJ, 536, L35

Lépine, S., Shara, M. M., \& Rich, R. M. 2002, AJ, 124, 1190

Lépine, S., Shara, M. M., \& Rich, R. M. 2003a, ApJ, 585, L69

Lépine, S., Rich, R. M., \& Shara, M. M. 2003b, ApJ, 591, L49

Lépine, S., Rich, R. M., \& Shara, M. M. 2003c, AJ, 125, 1598

Lépine, S., Shara, M. M., \& Rich, R. M. 2003d, AJ, 126, 921

Leonard, P. J. T., \& Tremaine, S. 1990, ApJ, 353, 486

Lodieu, N., Scholz, R.-D., \& McCaughrean, M. J. 2002, A\&A, 389, L20

Luyten, W. J. 1979, LHS Catalogue, Second Edition, (Minneapolis: Univ. Minnesota Press)

Luyten, W. J. 1979-1980, New Luyten Catalogue of Stars with Proper Motions Larger than Two Tenths of an Arcsecond. (Minneapolis: Univ. Minnesota), ADC Selected Astronomical Catalogs Vol. 1, CD-ROM

Martín, E. L., Delfosse, X., Basri, G., et al. 1999, AJ, 118, 2466

Martín, E. L. 2000, in Very low mass stars and brown dwarfs, ed. R. Rebolo, \& M. R. Zapatero Osorio (Cambridge: Cambridge University Press), 153

Meillon, L., Crifo, F., Gomez, A., Udry, S., \& Mayor, M. 1997, in Hipparcos Venice '97, ed. B. Battrick, ESA-SP, 402, 591

McCaughrean, M. J., Scholz, R.-D., \& Lodieu, N. 2002, A\&A, 390, L27

McCaughrean, M. J., Close, L. M., Scholz, R.-D., et al. 2004, A\&A, 413, 1029

Monet, D. G., Levine, S. E., Canzian, B., et al. 2003, AJ, 125, 984

Moorwood, A. F. M., \& Spyromilio, J. 1997, in The Early Universe with the VLT, ed. J. Bergeron (Heidelberg: Springer), 21

Phan-Bao, N., Crifo, F., Delfosse, X., et al. 2003, A\&A, 401, 959

Pokorny, R. S., Jones, H. R. A., \& Hambly, N. C. 2003, A\&A, 397, 575

Reid, I. N., Hawley, S. L., \& Gizis, J. E. 1995, AJ, 110, 1838

Reid, I. N., Kirkpatrick, J. D., Gizis, J. E., et al. 1999, ApJ, 527, L105

Reid, I. N., Kirkpatrick, J. D., Gizis, J. E., et al. 2000, AJ, 119, 369
Reid, I. N., Burgasser, A. J., Cruz, K. L., et al. 2001, AJ, 121, 1710

Reid, I. N., \& Cruz, K. L. 2002, AJ, 123, 2806

Reid, I. N., Kilkenny, D., \& Cruz, K. L. 2002a, AJ, 123, 2822

Reid, I. N., Kirkpatrick, J. D., Liebert, J., et al. 2002b, AJ, 124, 519

Reid, I. N., Cruz, K. L., Laurie, S. P., et al. 2003, AJ, 125, 354

Reylé, C., Robin, A. C., Scholz, R.-D., \& Irwin, M. J. 2002, A\&A, 390, 491

Ruiz, M. T., Leggett, S. K., \& Allard, F. 1997, ApJ, 491, 107

Ruiz, M. T., Wischnjewsky, M., Rojo, P. M., \& Gonzalez, L. E. 2001, ApJS, 133, 119

Salim, S., Lépine, S., Rich, R. M., \& Shara, M. M. 2003, ApJ, 586, 149

Scholz, R.-D., Irwin, M. J., Ibata, R., Jahreiß, H., \& Malkov, O. Yu. 2000, A\&A, 353, 958

Scholz, R.-D., Meusinger, H., \& Jahreiß, H. 2001, A\&A, 374, L12

Scholz, R.-D., Szokoly, G. P., Andersen, M., Ibata, R., \& Irwin, M. J. 2002, ApJ, 565, 539

Scholz, R.-D., \& Meusinger, H. 2002, MNRAS, 336, L49

Scholz, R.-D., McCaughrean, M. J., Lodieu, N., \& Kuhlbrodt, B. 2003, A\&A, 398, L29

Scholz, R.-D., Lodieu, N., Ibata, R., et al. 2004a, MNRAS, 347, 685

Scholz, R.-D., Lehmann, I., Matute, I., \& Zinnecker, H. 2004b, A\&A, 425,519

Scholz, R.-D., Lodieu, N., \& McCaughrean, M. J. 2004c, A\&A, in press

Schweitzer, A., Scholz, R.-D., Stauffer, J. R., Irwin, M. J., \& McCaughrean, M. J. 1999, A\&A, 350, L62

Teegarden, B. J., Pravdo, S. H., Hicks, M., et al. 2003, ApJ, 589, L51

Skrutskie, M. F., Schneider, S. E., Stiening, R., et al. 1997, The Impact of Large Scale Near-IR Sky Surveys, ed. F. Garzon et al. (Dordrecht: Kluwer), ASSL, 210, 25

Tinney, C. G. 1998, MNRAS, 296, L42

Tinney, C. G., Burgasser, A. J., \& Kirkpatrick, J. D. 2003, AJ, 126, 975

Tokunaga, A., \& Koyabashi, N. 1999, AJ, 117, 1010

Vrba, F. J., Henden, A. A., Luginbuhl, C. B., et al. 2004, AJ, 127, 2948

Wroblewski, H., \& Torres, C. 1994, A\&AS, 105, 179

Wroblewski, H., \& Costa, E. 1999, A\&AS, 139, 25

York, D. G., Adelman, J., Anderson, J. E., et al. 2000, AJ, 120, 1579

Zinnecker, H. 1995, in The bottom of the main sequence - and beyond, ed. C. G. Tinney (Heidelberg: Springer), 257 
N. Lodieu et al.: Spectroscopic classification of red high proper motion objects, Online Material p 1

\section{Online Material}


N. Lodieu et al.: Spectroscopic classification of red high proper motion objects, Online Material p 2

Table 1. List of new, red proper motion objects selected as explained in the text. Columns 1 and 2: reference number and name of the object; Cols. 3 and 4: equinox J2000 coordinates at the latest available epoch; Col. 5: epoch of position; Cols. 6-9: proper motion in mas $\mathrm{yr}^{-1}$ and associated errors; Cols. 10-12: $B_{J}, R, I$ magnitudes from SSS; Cols. 13-15: $J, H, K_{\mathrm{s}}$ magnitudes from 2MASS. Some comparison stars with well-determined spectral types are included in the list, namely LHS 517 (M3.5), 2MASS J0952-19 (M7.0), LP647-13 (M9), BRI B0021-0214 (M9.5), and Kelu 1 (L2.0). Notes ${ }^{a}$ and ${ }^{b}$ indicate that the object is also mentioned in the proper motion catalogue of Pokorny et al. (2003) and in the B1.0 catalogue (Monet et al. 2003), respectively.

\begin{tabular}{|c|c|c|c|c|c|c|c|c|c|c|c|c|c|}
\hline \multirow[t]{2}{*}{ RN Name } & \multicolumn{2}{|c|}{ Coordinates $(\mathrm{J} 2000)$} & \multirow[t]{2}{*}{ Epoch } & \multicolumn{4}{|c|}{ Proper Motions } & \multicolumn{6}{|c|}{ Optical and infrared magnitudes } \\
\hline & RA & Declination & & $\ell_{\alpha} \cos \delta$ & $\mu_{\delta}$ & $\sigma x$ & & $B_{J}$ & $R$ & $I$ & $J$ & $H$ & $K_{\mathrm{s}}$ \\
\hline $01 \mathrm{SSSP}$ & $05: 48.46$ & $21: 57: 19.7$ & 1999.60 & +721 & -128 & 7 & 2 & 22.56 & 19.41 & 16.21 & 13.27 & 12.62 & 12.20 \\
\hline 02 BRI B0021-0214 ${ }^{b}$ & $0: 24: 24.61$ & $-01: 58: 19.5$ & 2001.64 & -93 & +127 & 3 & 0 & 21.76 & 18.17 & 14.95 & 11.99 & 11.08 & 10.54 \\
\hline 03 SSSPM J0027-5402 ${ }^{b}$ & $0: 27: 23.43$ & $-54: 01: 46.1$ & 1999.80 & +415 & +41 & 18 & 4 & 19.15 & 18.06 & 14.70 & 12.36 & 11.72 & 11.34 \\
\hline 04 SSSPM J0030-3427 ${ }^{a}$ & $0: 30: 10.23$ & $-34: 26: 55.5$ & 2000.72 & -102 & -279 & 7 & 2 & 22.12 & 19.20 & 16.39 & 13.86 & 3.19 & 12.79 \\
\hline 05 LP $645-52^{b}$ & $0: 35: 41.68$ & $-03: 21: 30.8$ & 1998.71 & +428 & -84 & 3 & 1 & 20.26 & 17.88 & 15.54 & 13.75 & 13.28 & 12.99 \\
\hline 06 APMPM J00 & $0: 56: 53.38$ & $-76: 03: 43.3$ & 1998.60 & +160 & -389 & 4 & 13 & 19.88 & 16.91 & 14.61 & 12.57 & 11.99 & 11.62 \\
\hline $07 \mathrm{SSSP}$ & $9: 01.50$ & $-51: 00: 49.4$ & 1999.81 & +209 & +86 & 2 & 7 & 21.19 & 18.21 & 14.81 & 12.23 & 11.54 & 11.09 \\
\hline $08 \mathrm{SSSF}$ & 9:09.18 & $-49: 5$ & 1999.81 & +86 & +128 & 9 & 8 & 22.32 & 19.40 & 15.98 & 13.55 & 12.88 & 12.45 \\
\hline 09 LP $647-13^{b}$ & 17 & $-03: 43: 26.4$ & 1998.71 & +354 & +13 & 4 & 5 & 21.10 & 17.87 & 14.75 & 11.69 & 10.93 & 10.43 \\
\hline 10 SSSPM J0124-4240 & $1: 23: 59.05$ & $-42: 40: 07.3$ & 2000.63 & -145 & -229 & 4 & 7 & 22.16 & 19.35 & 16.17 & 13.15 & 12.47 & 12.04 \\
\hline $11 \mathrm{SSS}$ & $1: 24: 4$ & $-65: 46: 33.6$ & 1999.90 & +98 & +82 & 7 & 8 & 22.70 & 19.49 & 16.46 & 4.43 & 13.81 & 13.46 \\
\hline 12 SSSPI & $1: 33: 32.44$ & $-63:$ & 1999.90 & +77 & -81 & 8 & 9 & 22.11 & 19.01 & 15.97 & 14.51 & 14.02 & 13.70 \\
\hline 13 LP 769- & 1:59:17.41 & $-17: 30: 08.7$ & 2000.81 & -119 & -145 & 3 & 6 & 21.15 & 18.63 & 16.44 & 14.61 & 13.96 & 13.70 \\
\hline 14 SSSPI & $2: 04: 22.13$ & $-36: 32: 30.8$ & 2000.72 & +216 & -59 & 9 & 26 & & 19.96 & 15.77 & 13.27 & 12.60 & 12.19 \\
\hline $15 \mathrm{APM}$ & $2: 07: 05.28$ & $-72: 14: 06.9$ & 1999.86 & +282 & -120 & 6 & 5 & 21.07 & 18.59 & 17.40 & 16.50 & 15.93 & 15.82 \\
\hline 16 APN & $: 14.08$ & $-37:$ & 2000.72 & +422 & +134 & 2 & 6 & 20.79 & 18.10 & 14.91 & 12.44 & 11.83 & 11.38 \\
\hline $17 \mathrm{SSSH}$ & & -48 & & +118 & -332 & 9 & 5 & 22.20 & 19.14 & & & 12.96 & 12.52 \\
\hline 18 & & & & 95 & -174 & 4 & 5 & & & & & & \\
\hline $19 \mathrm{~S}$ & 4 & -54 & 2 & 07 & -14 & 7 & 8 & & 0 & & & 22 & 2.66 \\
\hline $20 \mathrm{SSSP}$ & $2: 31: 22.25$ & -41 & 1999.66 & +301 & -127 & 4 & 28 & & 20.10 & 17.03 & 13.85 & 13.27 & 12.89 \\
\hline 21 APMP & $2: 32: 08.96$ & $-44: 37: 00.1$ & 1998.92 & +384 & +284 & 5 & 5 & 21.42 & 19.00 & 16.56 & 14.45 & 13.82 & 13.74 \\
\hline 22 APM & $2: 44: 00.15$ & -52 : & 1999.77 & +305 & -11 & 7 & 3 & 21.49 & 18.96 & 17.23 & 15.92 & 15.41 & 14.99 \\
\hline $23 \mathrm{SSSF}$ & 3:06:11.59 & :52.8 & 2000.00 & -180 & -670 & 9 & 8 & 20.55 & 17.67 & 13.80 & 11.69 & 11.07 & 10.63 \\
\hline 24 SSSPN & 6:32.78 & 08.3 & 2000.77 & +286 & -22 & 25 & 28 & & 20.53 & 16.92 & 14.22 & 13.61 & 13.21 \\
\hline 25 APM & 10 & $-23:$ & 1998.89 & +621 & +58 & 8 & 5 & 21.30 & 18.87 & 17.08 & 30 & 15.31 & 14.94 \\
\hline 26 LP 88 & & $-30: 42: 38.8$ & & +40 & -392 & 5 & 6 & 19.91 & 17.21 & & & 10.70 & 10.26 \\
\hline 27 LP 944 & $3: 39: 35.21$ & $: 44.0$ & 1998.93 & +302 & +280 & 6 & 10 & 20.24 & 16.84 & 13.29 & & 10.02 & 9.55 \\
\hline $28 \mathrm{LP}^{7}$ & $: 35: 16.12$ & 57.4 & 1998.90 & +156 & +315 & 3 & 4 & 18.85 & 16.34 & 12.35 & 10.41 & 9.78 & 9.35 \\
\hline 29 LP 6 & 33 & -05 & 2001.79 & +339 & +126 & 2 & 2 & 18.85 & 16.50 & 13.17 & 10.66 & 9.99 & 9.55 \\
\hline 30 SSSPI & 77 & 27.3 & 1999.84 & +207 & -1022 & 9 & 2 & 20.10 & 17.31 & 15.56 & 14.44 & 14.12 & 13.97 \\
\hline $31 \mathrm{SSSP}$ & $5: 11: 01.63$ & $-46: 06: 01.5$ & 1999.77 & +53 & +121 & 9 & 6 & & 19.97 & 17.01 & 13.89 & 13.19 & 12.71 \\
\hline 32 APMF & $5: 36: 21.04$ & $-53: 58: 29.7$ & 1999.88 & +289 & -243 & 5 & 4 & 21.64 & 19.02 & 16.01 & 13.93 & 13.30 & 12.90 \\
\hline $33 \mathrm{ESO} 2$ & & $-49: 00: 50.3$ & 2000.15 & -34 & +401 & 14 & 8 & & 19.48 & 16.17 & & 12.54 & 12.10 \\
\hline 34 SSSPM J082 & $8: 28: 34.11$ & $-13: 09: 20.1$ & 2001.28 & -593 & +14 & 6 & 7 & 22.58 & 18.84 & 16.01 & 12.80 & 11.85 & 11.30 \\
\hline 35 LP $314-67^{b}$ & 9:48:05.16 & $+26: 24: 18.9$ & 1999.07 & -143 & -438 & 13 & 2 & & 18.03 & & 15.59 & 15.03 & 14.8 \\
\hline 36 2MASSW J0 & $9: 52: 21.88$ & $-19: 24: 31.9$ & 1998.32 & -76 & -100 & 3 & 2 & 19.37 & 17.02 & 13.84 & 11.86 & 11.26 & 10.87 \\
\hline 37 LP 61 & 12:07:51.63 & $-00: 52: 32.0$ & 1999.07 & -188 & -11 & 2 & 4 & 20.92 & 18.35 & 17.40 & 16.15 & 15.50 & 15.30 \\
\hline 38 APMP & $12: 11: 31.86$ & $-21: 25: 43.4$ & 1998.29 & -330 & -68 & 8 & 8 & 21.07 & 18.59 & 17.11 & 16.27 & 15.71 & 15.60 \\
\hline 39 APMPM J1222-2452 & $12: 22: 26.55$ & $-24: 52: 15.8$ & 1998.50 & -442 & +96 & 12 & 4 & 21.17 & 18.70 & 16.13 & 14.33 & 13.85 & 13.49 \\
\hline 40 LHS $2555 a^{b}$ & $12: 24: 46.13$ & $-32: 00: 16.8$ & 1999.26 & -598 & -243 & 12 & 9 & 19.54 & 17.29 & 15.73 & 14.31 & 13.79 & 13.57 \\
\hline 41 APMPM J1251-2121 & $12: 50: 52.65$ & $-21: 21: 13.6$ & 2000.20 & +435 & -349 & 4 & 3 & 19.40 & 17.03 & 13.64 & 11.16 & 10.55 & 10.13 \\
\hline 42 Kelu $1^{b}$ & 13:05:40.19 & $-25: 41: 05.9$ & 1998.41 & -322 & -19 & 10 & 5 & & 19.58 & 17.11 & 13.41 & 12.39 & 11.75 \\
\hline
\end{tabular}


N. Lodieu et al.: Spectroscopic classification of red high proper motion objects, Online Material p 3

Table 1. continued.

\begin{tabular}{|c|c|c|c|c|c|c|c|c|c|c|c|c|c|}
\hline \multirow[t]{2}{*}{ RN Name } & \multicolumn{2}{|c|}{ Coordinates (J2000) } & \multirow[t]{2}{*}{ Epoch } & \multicolumn{4}{|c|}{ Proper Motions } & \multicolumn{6}{|c|}{ Optical and infrared magnitudes } \\
\hline & RA & Declin & & $\mu_{\alpha} \cos \delta$ & $\mu_{\delta}$ & $\sigma x$ & $\sigma y$ & $B_{J}$ & $R$ & $I$ & $J$ & $H$ & $K_{\mathrm{s}}$ \\
\hline 43 CE $303^{b}$ & $: 09: 21.85$ & $-23: 30: 35.0$ & 32 & +8 & -376 & 9 & 2 & 20.41 & 17.83 & $\overline{52}$ & 8 & .08 & 0.67 \\
\hline $44 \mathrm{CE} 352^{b}$ & $40: 3$ & -30 & 2000.23 & -335 & -103 & 7 & 4 & 20.58 & .26 & 7.23 & 5.70 & 5.27 & 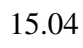 \\
\hline 45 LP $859-1^{b}$ & 04: & -23 & 0. & -339 & -85 & 14 & 3 & 20.26 & 17.82 & 4.66 & 12.01 & 38 & 11.0 \\
\hline 46 LHS $3141 \mathrm{~B}^{b}$ & $: 59: 3$ & -22 & 1999.32 & +193 & -538 & 8 & 21 & 21.65 & 19.41 & 16.34 & 13.61 & 13.12 & 12.84 \\
\hline 47 SSSPM J1 & $19: 26: 08.59$ & $-43:$ & 1999.53 & -167 & -1072 & 15 & 20 & 18.75 & 16.69 & 13.65 & 11.94 & 11.42 & 11.12 \\
\hline 48 APMPM J1957-4216 ${ }^{b}$ & $19: 56: 57.61$ & $-42: 16: 23.5$ & 2000.57 & +149 & -1017 & 4 & 6 & 19.39 & 17.32 & 14.53 & 12.38 & 11.99 & 11.66 \\
\hline 49 LP $815-21^{b}$ & $20: 28: 04.52$ & $-18: 18: 57.5$ & 1998.44 & -105 & -175 & 5 & & 20.38 & 18.11 & 17.45 & 16.10 & 15.91 & 15.28 \\
\hline 50 SSSPM J2 & $20: 32: 32.91$ & $-69: 18: 59.1$ & 2000.43 & +228 & -459 & 10 & 5 & 22.59 & 19.75 & 16.42 & 13.64 & 12.98 & 12.58 \\
\hline 51 APMPM J & $20: 35: 49.96$ & $: 07.7$ & 1999.71 & -86 & -416 & 4 & 8 & 21.17 & 18.75 & 16.34 & 14.62 & 14.16 & 13.75 \\
\hline 52 SSSPM J & $20: 52: 2$ & -47 & 78 & -7 & -435 & 5 & 7 & 21.67 & 18.82 & 15.55 & 12.94 & 12.29 & 11.88 \\
\hline $53 \mathrm{~S}$ & 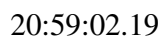 & -80 & 20 & +361 & -46 & 6 & 29 & & 20.50 & 16.91 & 14.28 & 13.69 & 10.4 \\
\hline $54 \mathrm{~S}$ & 21.01 & -51 & & +69 & -123 & 7 & 16 & & 20.27 & 17.09 & 15.13 & & 14.09 \\
\hline $55[\mathrm{HB} 88] \mathrm{N}$ & & -45 : & & +388 & -475 & 14 & 15 & 22.36 & 17.41 & & 13.43 & & 12.37 \\
\hline $56[\mathrm{HB} 88] \mathrm{N}$ & 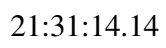 & -42 & 3 & +48 & -71 & 6 & 9 & 7 & 3 & 16 & 14.52 & & 13.57 \\
\hline 57 LP 819-9 ${ }^{b}$ & 2 & -15 & 8 & +0 & -283 & 2 & 4 & 20.80 & 18.47 & 16.50 & 15.07 & 59 & 14.31 \\
\hline 58 LHS $517^{b}$ & 22:09: & 26.7 & 1999.39 & +1125 & -24 & 2 & 1 & 11.64 & 9.35 & 7.55 & 6.51 & 5.90 & 5.59 \\
\hline 59 SSSPM J2229 & $22: 29: 23.65$ & -69 & 2000.58 & +47 & -217 & 9 & 10 & & 19.83 & 16.97 & 14.47 & 13.76 & 13.35 \\
\hline 60 LDS4980 & $22: 35: 5$ & +07 & 2000.59 & -112 & -208 & 2 & & & 18.35 & & 15.05 & 14.53 & 14.25 \\
\hline $61 \mathrm{LD}$ & $22: 36: 00.63$ & 03.5 & 2000.59 & -103 & -210 & 9 & & & 17.54 & & 14.65 & 14.26 & 13.86 \\
\hline $62 \mathrm{SS}$ & $22: 40: 26.97$ & -42 & 2000.73 & -35 & -539 & 27 & 9 & & 19.67 & 16.53 & 13.76 & 13.19 & 12.80 \\
\hline $63 \mathrm{SSSP}$ & $22: 57:$ & -52 & 1999.82 & +57 & +113 & 11 & 16 & & 0.20 & 17.33 & 14.93 & 14.37 & 14.00 \\
\hline 64 SSS & 22 & -4 & 20 & -185 & +197 & 11 & 4 & & 1 & 3 & 1 & & 12.60 \\
\hline 65 SSSPM J23 & & & & +452 & +25 & 10 & 6 & 22.80 & 19.70 & 16.72 & 13.39 & & 12.24 \\
\hline $66 \mathrm{SSS}$ & . & 9.0 & 1998.50 & -23 & -271 & 36 & 24 & & 20.52 & 17.68 & 14.38 & & 12.97 \\
\hline 67 SSSPI & .18 & -4 & 200 & +216 & -16 & 9 & 3 & 22.12 & 19.25 & 16.22 & 13.76 & 13 & 12.68 \\
\hline $68 \mathrm{SSSP}$ & $23: 22: 0$ & -63 & 1999.88 & +121 & -19 & 11 & 5 & & 19.54 & 16.48 & 14.26 & 13.65 & 13.20 \\
\hline 69 APM & $23: 30: 16.12$ & -47 & 2000.79 & -578 & -983 & 2 & 2 & 19.38 & 16.79 & 13.29 & 11.23 & 10.64 & 10.28 \\
\hline 70 APN & $23: 31: 21.74$ & -27 & 1999.44 & +85 & +753 & 4 & 3 & 20.42 & 17.89 & 14.41 & 11.65 & 11.06 & 10.65 \\
\hline 71 SSSPM J2335-6913 & $23: 35: 19.59$ & $-69: 13: 17.0$ & 2000.76 & +66 & -168 & 10 & 3 & 22.25 & 19.29 & 16.46 & 13.92 & 13.25 & 12.90 \\
\hline 72 AРMPM J2344-2906 & $23: 43: 31.98$ & $-29: 06: 27.1$ & 1998.85 & +331 & -217 & 10 & 6 & 21.12 & 18.64 & 15.34 & 13.26 & 12.75 & 12.43 \\
\hline 73 SSSPM J2345-6810 & 23:44:57.97 & $-68: 09: 39.8$ & 2000.77 & +206 & -81 & 11 & 4 & 22.26 & 19.49 & 16.39 & 13.98 & 13.36 & 12.96 \\
\hline 74 AРMPM J2347-3154 & $23: 46: 54.71$ & $-31: 53: 53.2$ & 1998.95 & +424 & -408 & 3 & 4 & 22.51 & 19.49 & 15.91 & 13.28 & 12.68 & 12.20 \\
\hline 75 SSSPM J2 & $23: 51: 50.44$ & -25 : & 1999.60 & +354 & +193 & 9 & 8 & 21.73 & 18.70 & 15.27 & 12.47 & 11.73 & 11.27 \\
\hline $76 \mathrm{SS}$ & 23:53:01.41 & $-41: 23: 24.6$ & 1999.70 & +130 & +0 & 20 & 8 & 22.23 & 19.08 & 16.89 & 14.39 & 13.73 & 13.33 \\
\hline $2354-3316^{a, b}$ & 23:54:09.28 & $-33: 16: 26.6$ & 1999.57 & -326 & -389 & 8 & 13 & 22.20 & 19.30 & 16.57 & 13.05 & 12.36 & 11.88 \\
\hline 78 SSSPM J2356-3426 & $: 10.81$ & $-34: 26: 04.4$ & 199 & +70 & -301 & 9 & 5 & 22.21 & 19.18 & 16.15 & 12.95 & 12.38 & 11.97 \\
\hline 79 SSSPM J2400-2008 & 23:59:57.62 & $-20: 07: 39.4$ & 1998.61 & +402 & -511 & 29 & 10 & & 20.29 & 17.45 & 14.38 & 13.62 & 13.25 \\
\hline
\end{tabular}


N. Lodieu et al.: Spectroscopic classification of red high proper motion objects, Online Material p 4

Table 2. Observing logs for the subset of proper motion objects observed photometrically with FORS1 $(R$ and $I)$ and ISAAC $\left(J_{\mathrm{s}}, H, K_{\mathrm{s}}\right)$ mounted on the VLT. Names, observing dates, and exposure times for the optical and near-infrared observations are given for each individual object.

\begin{tabular}{|c|c|c|c|c|c|c|c|c|c|c|c|}
\hline \multirow[t]{2}{*}{$\mathrm{RN}$} & \multirow[t]{2}{*}{ Name } & \multicolumn{5}{|c|}{ Optical imaging } & \multicolumn{5}{|c|}{ Near-infrared imaging } \\
\hline & & Date & $\operatorname{ExpT}(R)$ & $R$ & $\operatorname{ExpT}(I)$ & $I$ & Date & ExpT & $J_{\mathrm{s}}$ & $H$ & $K_{\mathrm{s}}$ \\
\hline 02 & BRI B0021-0214 & 1999 Jun. 01 & $3 \times 10 \mathrm{~s}$ & 17.42 & $3 \times 5 s$ & 15.34 & 1999 Jul. 22 & $3 \times 2 \mathrm{~s}$ & 11.87 & 11.16 & 10.60 \\
\hline 05 & LP $645-52$ & 1999 Jun. 01 & $3 \times 10 \mathrm{~s}$ & 17.56 & $3 \times 5 s$ & 15.73 & 1999 Jun. 02 & $3 \times 2 s$ & 13.80 & 13.35 & 13.05 \\
\hline 06 & APMPM J0057-7604 & 2000 Jun. 21 & $3 \times 10 \mathrm{~s}$ & 16.63 & $3 \times 5 \mathrm{~s}$ & 14.28 & 2000 Jun. 19 & $3 \times 2 \mathrm{~s}$ & 12.71 & 12.09 & 11.75 \\
\hline 13 & LP 769-14 & 1999 Jul. 25 & $3 \times 10 \mathrm{~s}$ & 18.20 & $3 \times 5 s$ & 16.50 & 1999 Jun. 02 & $3 \times 2 s$ & 14.62 & 14.05 & 13.74 \\
\hline 15 & APMPM J0207-7214 & 1999 Jun. 01 & $3 \times 10 \mathrm{~s}$ & 18.50 & $3 \times 5 s$ & 17.70 & 1999 Jul. 23 & $3 \times 2 s$ & 16.42 & 15.95 & 15.75 \\
\hline 16 & APMPM J0207-3722 & 1999 Jul. 25 & $3 \times 10 s$ & 17.44 & $3 \times 5 \mathrm{~s}$ & 15.24 & 1999 Jul. 23 & $3 \times 2 \mathrm{~s}$ & 12.48 & 11.86 & 11.44 \\
\hline 21 & APMPM J0232-4437 & 2000 Jun. 21 & $3 \times 10 \mathrm{~s}$ & 18.40 & $3 \times 5 \mathrm{~s}$ & 16.04 & 2000 Jun. 19 & $3 \times 2 \mathrm{~s}$ & 14.65 & 14.13 & 13.79 \\
\hline 22 & APMPM J0244-5203 & 2000 Jun. 21 & $3 \times 10 \mathrm{~s}$ & 18.68 & $3 \times 5 s$ & 16.86 & 2000 Jun. 22 & $3 \times 2 \mathrm{~s}$ & 16.11 & 15.59 & 15.26 \\
\hline 25 & APMPMJ0331-2349 & 1999 Aug. 29 & $3 \times 10 \mathrm{~s}$ & 18.50 & $3 \times 5 s$ & 17.00 & 1999 Sep. 06 & $3 \times 2 s$ & 15.64 & 15.30 & 15.18 \\
\hline 32 & APMPM J0536-5358 & 2000 Apr. 08 & $3 \times 10 s$ & 18.20 & $3 \times 5 \mathrm{~s}$ & 16.50 & 2000 Aug. 05 & $3 \times 2 \mathrm{~s}$ & 14.02 & 13.41 & 13.02 \\
\hline 35 & LP 314-67 & 1999 Apr. 23 & $3 \times 10 \mathrm{~s}$ & 18.10 & $3 \times 5 \mathrm{~s}$ & 17.00 & 2000 May 12 & $3 \times 2 \mathrm{~s}$ & - & - & - \\
\hline 37 & LP 614-35 & 2000 Apr. 04 & $3 \times 10 \mathrm{~s}$ & 18.47 & $\mathrm{~s}$ & 17.42 & 1999 May 29 & $3 \times 2 s$ & 16.19 & 15.58 & 15.39 \\
\hline 38 & APMPM J1212-2126 & 2000 Apr. 04 & $3 \times 10 \mathrm{~s}$ & 18.41 & $3 \times 5 s$ & 17.36 & 2000 May 15 & $3 \times 2 s$ & 16.26 & 15.81 & 15.69 \\
\hline 39 & APMPM J1222-2452 & 2000 Apr. 04 & $3 \times 10 s$ & 18.17 & $3 \times 5 \mathrm{~s}$ & 16.31 & 2000 May 15 & $3 \times 2 \mathrm{~s}$ & 14.38 & 13.94 & 13.64 \\
\hline 40 & LHS 2555a & 2000 Apr. 04 & $3 \times 10 \mathrm{~s}$ & 16.92 & $3 \times 5 s$ & 15.64 & 2000 May 15 & $3 \times 2 \mathrm{~s}$ & 14.37 & 13.91 & 13.70 \\
\hline 41 & APMPM J1251-2121 & 2000 Apr. 04 & $3 \times 10 \mathrm{~s}$ & 16.07 & $3 \times 5 s$ & 13.95 & 2000 May 15 & $3 \times 2 s$ & 11.25 & 10.67 & 10.29 \\
\hline 42 & Kelu 1 & 1999 Apr. 23 & $3 \times 10 \mathrm{~s}$ & 19.10 & $3 \times 5 s$ & 17.20 & 2000 Oct. 25 & $3 \times 2 s$ & 13.29 & 12.42 & 11.73 \\
\hline 43 & CE 303 & 2000 Apr. 02 & $3 \times 10 \mathrm{~s}$ & 17.00 & $3 \times 5 \mathrm{~s}$ & 14.77 & 2000 May 15 & $3 \times 2 \mathrm{~s}$ & 11.88 & 11.31 & 10.88 \\
\hline 44 & CE 352 & 1999 Apr. 23 & $3 \times 10 \mathrm{~s}$ & 18.10 & $3 \times 5 s$ & 17.10 & 1999 Apr. 30 & $3 \times 2 s$ & 15.77 & 15.29 & 15.10 \\
\hline 45 & LP 859-1 & 2000 Apr. 02 & $3 \times 10 \mathrm{~s}$ & 16.96 & $3 \times 5 \mathrm{~s}$ & 14.77 & 2000 Apr. 24 & $3 \times 2 \mathrm{~s}$ & 12.16 & 11.58 & 11.16 \\
\hline 46 & LHS 3141B & 2000 Apr. 02 & $3 \times 10 \mathrm{~s}$ & 18.46 & $3 \times 5 \mathrm{~s}$ & 16.15 & 2000 Apr. 24 & $3 \times 2 s$ & 13.71 & 13.26 & 13.12 \\
\hline 49 & LP 815-21 & 1999 Мay 31 & $3 \times 10 s$ & 18.11 & $3 \times 5 \mathrm{~s}$ & 17.37 & 2000 Oct. 25 & $3 \times 2 \mathrm{~s}$ & 16.12 & 15.64 & 15.39 \\
\hline 51 & APMPM J20 & 2000 Apr. 02 & $3 \times 10 \mathrm{~s}$ & 18.51 & $3 \times 5 s$ & 16.53 & 2000 May 15 & $3 \times 2 s$ & 14.72 & 14.29 & 13.98 \\
\hline 56 & [HB88] M12 & 1999 May 31 & $3 \times 10 \mathrm{~s}$ & 18.76 & $3 \times 5 s$ & 16.58 & 2000 Oct. 25 & $3 \times 2 s$ & 14.51 & 13.92 & 13.58 \\
\hline 57 & LP 819-9 & 1999 May 31 & $3 \times 10 s$ & 18.10 & $3 \times 5 \mathrm{~s}$ & 16.65 & 1999 May 29 & $3 \times 2 \mathrm{~s}$ & 15.05 & 14.60 & 14.32 \\
\hline 60 & LDS4980 B & 1999 May 31 & $3 \times 10 \mathrm{~s}$ & 18.33 & $3 \times 5 \mathrm{~s}$ & 16.75 & 1999 Jun. 02 & $3 \times 2 \mathrm{~s}$ & 14.66 & 14.16 & 13.88 \\
\hline 61 & LDS4980 A & 1999 May 31 & $3 \times 40 \mathrm{~s}$ & 18.09 & $3 \times 10 \mathrm{~s}$ & 16.35 & 2000 Oct. 25 & $3 \times 2 \mathrm{~s}$ & - & - & - \\
\hline 69 & APMPM J2330-4737 & 1999 Jun. 01 & $3 \times 40 \mathrm{~s}$ & 15.91 & $3 \times 10 \mathrm{~s}$ & 13.78 & 2000 Sep. 22 & $3 \times 2 s$ & 11.27 & 10.73 & 10.37 \\
\hline 70 & APMPM J2331-2750 & 1999 Jun. 01 & $3 \times 40 \mathrm{~s}$ & 16.68 & $3 \times 10 \mathrm{~s}$ & 14.45 & 2000 Oct. 25 & $3 \times 2 \mathrm{~s}$ & 11.67 & 11.09 & 10.73 \\
\hline 72 & APMPM J2344-2906 & 1999 Jun. 01 & $3 \times 40 s$ & 17.96 & $3 \times 10 \mathrm{~s}$ & 15.71 & 1999 Jul. 22 & $3 \times 2 s$ & 13.19 & 12.78 & 12.42 \\
\hline 74 & APMPM J2347-3154 & 2000 May 12 & $3 \times 40 s$ & 18.45 & $3 \times 10 \mathrm{~s}$ & 16.20 & 2000 Jun. 19 & $3 \times 2 \mathrm{~s}$ & 13.75 & 12.82 & 12.34 \\
\hline 77 & APMPM J2354-3316 & 2000 May 12 & $3 \times 40 s$ & 18.36 & $3 \times 10 \mathrm{~s}$ & 16.15 & 2000 May 15 & $3 \times 2 s$ & 13.21 & 12.61 & 12.10 \\
\hline
\end{tabular}


N. Lodieu et al.: Spectroscopic classification of red high proper motion objects, Online Material p 5

Table 3. Observing logs for the first set of proper motion objects observed spectroscopically with VLT/FORS1 and VLT/ISAAC in the optical and near-infrared, respectively. Names, observing dates, and exposure times are provided for both wavelength ranges. All optical spectra were obtained with Grism_300I except for APMPM J2036-4936 and LP 859-1 which were observed with Grism_150I. The near-infrared spectra of the two optically classified extreme subdwarfs LP 815-21 and CE 352 were noisy and unusable, and therefore are not shown in Fig. 6.

\begin{tabular}{|c|c|c|c|c|c|c|c|}
\hline \multirow{2}{*}{\multicolumn{2}{|c|}{ RN Name }} & \multicolumn{2}{|c|}{ Optical spectroscopy } & \multicolumn{4}{|c|}{ Near-infrared spectroscopy } \\
\hline & & Date & ExpT & Date & $\operatorname{ExpT}(1.10-1.39 \mu \mathrm{m})$ & $\operatorname{ExpT}(1.42-1.83 \mu \mathrm{m})$ & $\operatorname{ExpT}(1.84-2.56 \mu \mathrm{m})$ \\
\hline 02 & BRI B0021-0214 & 1999 Aug. 31 & $3 \times 100 \mathrm{~s}$ & - & - & - & - \\
\hline 05 & LP 645-52 & 1999 Aug. 31 & $3 \times 100 s$ & - & - & - & - \\
\hline 13 & LP 769-14 & 2000 Jun. 21 & $5 \times 100 \mathrm{~s}$ & - & - & - & - \\
\hline 16 & APMPM J0207-3722 & 1999 Aug. 29 & $3 \times 100 \mathrm{~s}$ & - & - & - & - \\
\hline 35 & LP 314-67 & 2000 Apr. 05 & $3 \times 100 \mathrm{~s}$ & - & - & - & - \\
\hline 37 & LP 614-35 & 2000 Apr. 04 & $3 \times 250 \mathrm{~s}$ & - & - & - & - \\
\hline 39 & APMPM J1222-2452 & 2000 Jun. 21 & $5 \times 100 \mathrm{~s}$ & - & - & - & - \\
\hline 41 & APMPM J1251-2121 & 2000 Jun. 21 & $3 \times 100 \mathrm{~s}$ & - & - & - & - \\
\hline 42 & Kelu 1 & 1999 Jul. 07 & $3 \times 250 \mathrm{~s}$ & 1999 Jul. 27 & $4 \times 50 \mathrm{~s}$ & $4 \times 50 s$ & $6 \times 50 s$ \\
\hline 43 & CE 303 & 2000 Jun. 21 & $3 \times 100 s$ & - & - & - & - \\
\hline 44 & CE 352 & 1999 Jul. 07 & $3 \times 100 \mathrm{~s}$ & 1999 Jul. 28 & $10 \times 100 \mathrm{~s}$ & $10 \times 100 \mathrm{~s}$ & $18 \times 100 \mathrm{~s}$ \\
\hline 45 & LP 859-1 & 2000 Apr. 24 & $3 \times 100 \mathrm{~s}$ & - & - & - & - \\
\hline 46 & LHS 3141B & 2000 May 06 & $5 \times 250 \mathrm{~s}$ & - & - & - & - \\
\hline 49 & LP $815-21$ & 1999 Aug. 27 & $3 \times 250 \mathrm{~s}$ & 1999 Jul. 06 & $5 \times 100 \mathrm{~s}$ & $5 \times 100 \mathrm{~s}$ & $18 \times 100 \mathrm{~s}$ \\
\hline 51 & APMPM J2036-4936 & 1999 Aug. 27 & $3 \times 250 \mathrm{~s}$ & - & - & - & - \\
\hline 56 & [HB88] M12 & 1999 Aug. 26 & $3 \times 250 \mathrm{~s}$ & 1999 Jul. 06 & $4 \times 100 \mathrm{~s}$ & $4 \times 100 \mathrm{~s}$ & $6 \times 100 \mathrm{~s}$ \\
\hline 57 & LP 819-9 & 1999 Aug. 29 & $3 \times 250 \mathrm{~s}$ & - & - & - & - \\
\hline 60 & LDS4980 B & 1999 Aug. 29 & $3 \times 250 \mathrm{~s}$ & - & - & - & - \\
\hline 61 & LDS4980 A & 1999 Aug. 29 & $3 \times 250 \mathrm{~s}$ & - & - & - & - \\
\hline 69 & APMPM J2330-4737 & 1999 Aug. 29 & $5 \times 100 \mathrm{~s}$ & - & - & - & - \\
\hline 70 & APMPM J2331-2750 & 1999 Aug. 28 & $6 \times 100 s$ & 1999 Jul. 23 & $4 \times 25 \mathrm{~s}$ & $4 \times 25 \mathrm{~s}$ & $6 \times 25 s$ \\
\hline 72 & APMPM J2344-2906 & 1999 Aug. 28 & $5 \times 100 \mathrm{~s}$ & 1999 Jul. 23 & $4 \times 25 \mathrm{~s}$ & $4 \times 25 \mathrm{~s}$ & $6 \times 25 s$ \\
\hline
\end{tabular}


Table 4. Observing logs for all proper motion targets observed in the optical with Grism\#12 in EFOSC2 on the ESO 3.6-m and in the nearinfrared with the Blue and Red gratings in SOFI on the NTT. Names, observing dates, and exposure times are provided for both wavelength ranges.

\begin{tabular}{|c|c|c|c|c|c|}
\hline $\mathrm{RN}$ & Name & Optical spe & troscopy & Near-infrared & ectroscopy \\
\hline & & Date & ExpT & Date & ExpT \\
\hline 01 & SSSPM J0006-2157 & 2001 Nov. 21 & $3 \times 1000 \mathrm{~s}$ & - & - \\
\hline 03 & SSSPM J0027-5402 & 2002 Dec. 06 & $1 \times 400 \mathrm{~s}$ & - & - \\
\hline 04 & SSSPM J0030-3427 & - & - & 2001 Nov. 25 & $3 \times 150 \mathrm{~s}$ \\
\hline 07 & SSSPM J0109-5101 & 2002 Dec. 05 & $3 \times 400 \mathrm{~s}$ & 2001 Nov. 25 & $3 \times 60 \mathrm{~s}$ \\
\hline 08 & SSSPM J0109-4955 & - & - & 2001 Nov. 25 & $3 \times 150 \mathrm{~s}$ \\
\hline 09 & LP 647-13 & 2002 Dec. 05 & $1 \times 360 \mathrm{~s}$ & - & - \\
\hline 10 & SSSPM J0124-4240 & - & - & 2001 Nov. 25 & $3 \times 150 \mathrm{~s}$ \\
\hline 11 & SSSPM J0125-6546 & - & - & 2001 Nov. 25 & $3 \times 200 s$ \\
\hline 12 & SSSPM J0134-6315 & - & - & 2001 Nov. 25 & $3 \times 150 \mathrm{~s}$ \\
\hline 14 & SSSPM J0204-3633 & 2001 Nov. 22 & $1 \times 500 \mathrm{~s}$ & 2001 Nov. 24 & $3 \times 200 s$ \\
\hline 16 & APMPM J0207-3722 & - & - & 2001 Nov. 24 & $3 \times 150 \mathrm{~s}$ \\
\hline 17 & SSSPM J0215-4804 & - & - & 2001 Nov. 25 & $3 \times 150 \mathrm{~s}$ \\
\hline 18 & SSSPM J0219-1939 & 2001 Nov. 21 & $3 \times 900 \mathrm{~s}$ & 2001 Nov. 24 & $3 \times 250 \mathrm{~s}$ \\
\hline 19 & SSSPM J0222-5412 & 2002 Dec. 08 & $2 \times 900 \mathrm{~s}$ & - & - \\
\hline 20 & SSSPM J0231-4122 & 2002 Dec. 08 & $1 \times 800 \mathrm{~s}$ & - & - \\
\hline 23 & SSSPM J0306-3648 & 2001 Nov. 22 & $1 \times 300 \mathrm{~s}$ & 2001 Nov. 24 & $3 \times 120 \mathrm{~s}$ \\
\hline 24 & SSSPM J0327-4236 & 2002 Dec. 06 & $1 \times 900 \mathrm{~s}$ & - & - \\
\hline 26 & LP 888-18 & 2001 Nov. 22 & $1 \times 270 \mathrm{~s}$ & 2001 Nov. 24 & $3 \times 100 s$ \\
\hline 27 & LP 944-20 & 2001 Nov. 22 & $1 \times 240 \mathrm{~s}$ & 2001 Nov. 24 & $3 \times 60 s$ \\
\hline 28 & LP 775-31 & 2001 Nov. 22 & $1 \times 200 \mathrm{~s}$ & 2001 Nov. 24 & $3 \times 60 \mathrm{~s}$ \\
\hline 29 & LP $655-48$ & 2001 Nov. 22 & $1 \times 200 \mathrm{~s}$ & 2001 Nov. 24 & $3 \times 60 s$ \\
\hline 30 & SSSPM J05 & 2001 Nov. 22 & $1 \times 300 \mathrm{~s}$ & - & - \\
\hline 31 & SSSPM J0511-4606 & 2002 Dec. 08 & $1 \times 780 \mathrm{~s}$ & - & - \\
\hline 33 & ESO 207-61 & 2002 Dec. 06 & $2 \times 600 \mathrm{~s}$ & - & - \\
\hline 34 & SSSPM J0829-1309 & 2002 Dec. 08 & $1 \times 480 \mathrm{~s}$ & - & - \\
\hline 36 & 2MASS J0952-19 & 2001 Nov. 22 & $1 \times 240 \mathrm{~s}$ & - & - \\
\hline 47 & SSSPM J1926-4311 & 2002 Dec. 05 & $1 \times 240 \mathrm{~s}$ & - & - \\
\hline 48 & APMPM J1957-4216 & 2002 Dec. 06 & $1 \times 240 \mathrm{~s}$ & - & - \\
\hline 50 & SSSPM J2033-6919 & 2001 Nov. 21 & $3 \times 1200 \mathrm{~s}$ & - & - \\
\hline 52 & SSSPM J2052-4759 & 2002 Dec. 05 & $1 \times 540 \mathrm{~s}$ & - & - \\
\hline 53 & SSSPM J2059-8018 & 2001 Nov. 22 & $2 \times 900 \mathrm{~s}$ & - & - \\
\hline 55 & [HB88] M18 & 2002 Dec. 06 & $2 \times 600 \mathrm{~s}$ & - & - \\
\hline 58 & LHS 517 & 2002 Dec. 08 & $1 \times 15 \mathrm{~s}$ & - & - \\
\hline 59 & SSSPM J2229-6931 & - & - & 2001 Nov. 25 & $3 \times 200 s$ \\
\hline 62 & SSSPM J2240-4253 & 2002 Dec. 06 & $1 \times 660 \mathrm{~s}$ & - & - \\
\hline 63 & SSSPM J2257-5208 & 2002 Dec. 08 & $1 \times 720 \mathrm{~s}$ & - & - \\
\hline 64 & SSSPM J2258-4639 & 2002 Dec. 08 & $1 \times 720 \mathrm{~s}$ & - & - \\
\hline 65 & SSSPM J2307-5009 & 2002 Dec. 06 & $1 \times 660 \mathrm{~s}$ & 2001 Nov. 25 & $3 \times 200 \mathrm{~s}$ \\
\hline 66 & SSSPM J2310-1759 & 2001 Nov. 22 & $3 \times 900 \mathrm{~s}$ & 2001 Nov. 24 & $3 \times 250 \mathrm{~s}$ \\
\hline 67 & SSSPM J2319-4919 & - & - & 2001 Nov. 25 & $3 \times 150 \mathrm{~s}$ \\
\hline 68 & SSSPM J2322-6358 & 2001 Nov. 22 & $1 \times 600 \mathrm{~s}$ & - & - \\
\hline 71 & SSSPM J2335-6913 & 2001 Nov. 22 & $1 \times 600 \mathrm{~s}$ & - & - \\
\hline 73 & SSSPM J2345-6810 & 2002 Dec. 08 & $1 \times 600 \mathrm{~s}$ & 2001 Nov. 25 & $3 \times 200 s$ \\
\hline 74 & APMPM J2347-3154 & 2001 Nov. 21 & $3 \times 1200 \mathrm{~s}$ & - & - \\
\hline 75 & SSSPM J2352-2538 & 2001 Nov. 22 & $1 \times 400 \mathrm{~s}$ & 2001 Nov. 24 & $3 \times 150 \mathrm{~s}$ \\
\hline 76 & SSSPM J2353-4123 & 2002 Dec. 06 & $1 \times 540 \mathrm{~s}$ & - & - \\
\hline 77 & APMPM J2354-3316 & 2002 Dec. 08 & $1 \times 540 \mathrm{~s}$ & 2001 Nov. 25 & $3 \times 150 \mathrm{~s}$ \\
\hline 78 & SSSPM J2356-3426 & 2002 Dec. 08 & $2 \times 500 \mathrm{~s}$ & 2001 Nov. 25 & $3 \times 150 \mathrm{~s}$ \\
\hline 79 & SSSPM J2400-2008 & 2002 Dec. 08 & $3 \times 900 \mathrm{~s}$ & 2001 Nov. 24 & $3 \times 250 \mathrm{~s}$ \\
\hline
\end{tabular}

